\title{
Modeling of progressive failures in quasi-brittle media based on a temporal stress-redistribution mechanism
}

\author{
Jin Xing Liu ${ }^{\mathrm{a}, *}$, Nai Gang Liang ${ }^{\mathrm{b}}$, Ai Kah Soh ${ }^{\mathrm{c}}$ \\ a Faculty of Civil Engineering and Mechanics, Jiangsu University, Xuefu Road 301, Zhenjiang 212013, Jiangsu, China \\ b State key laboratory of Nonlinear Mechanics, Institute of Mechanics, Chinese Academy of Sciences, Beijing 100190, China \\ ' School of Engineering, Monash University Malaysia, Jalan Lagoon Selatan, Bandar Sunway 47500, Selangor Darul Ehsan, Malaysia
}

\section{A R T I C L E I N F O}

\section{Article history:}

Received 9 December 2017

Revised 22 July 2018

Accepted 23 August 2018

Available online 6 September 2018

\section{Keywords:}

Progressive quasi-brittle failure

Stress redistribution

Damage-induced softening

Viscous force field

Event-driven algorithm

Strain rate sensitivity

\begin{abstract}
A B S T R A C T
A new attempt is made to simulate progressive failure processes in heterogeneous brittle materials such as concrete, ceramics, rocks etc., by considering the time-dependence of stress redistributions induced by local breakages. Two mechanisms of stress redistribution are incorporated into the proposed model in order to account for the influence of each local breakage on the remaining specimen: (1) one is the immediate release of internal forces in the breaking element, which is assumed to happen within an infinitesimal time when compared with the characteristic time of external loadings. The release of such internal forces is hence suddenly applied to the remaining specimen, which is considered to take time to deform correspondingly due to material viscosity. This deformation delay is implemented by introducing a viscous force (VF) field prevailing in the entire specimen. (2) The other is the gradual release of previously stored VF fields, whose characteristic time is assumed to be material-dependent. Here the release of VF is approximated as stepwise for simplicity. The proposed model is found to be capable of overcoming the unreasonablylow-ductility problem encountered in many existing lattice models when it comes to the uniaxial tensile test. Furthermore, the force-displacement response obviously depends on the ratio of the VF releasing time to the characteristic time of external loading, showing trends agreeing with experimental observations. Compared with results without viscosity, the failure pattern is more scattering, and the force-displacement curve has a higher peak load and a more ductile post-peak tail.
\end{abstract}

(c) 2018 Elsevier Inc. All rights reserved.

\section{Introduction}

Quasi-brittle materials can be natural or artificial, including but not limited to concrete, cementitious composites, sea-ice, cohesive and frozen soils, and toughened ceramics. In above media, cracks propagate progressively under external loadings, which, compared with ideally brittle materials like glass, has some distinctive features such as post-peak softening, and has attracted intensive research interests (e.g. [1-6]). Lattice-type models have been widely used to study such phenomena [715], in which the continuum-like material was considered as a network composed of particular fundamental elements such as bars, beams or various link elements, usually based on the principle of strain energy equivalence [11,13], or by installing relevant springs between each two neighboring rigid bodies [16,17].

\footnotetext{
* Corresponding author.

E-mail address: jxliu@mails.ucas.ac.cn (J.X. Liu).
} 
Failure happens when the deformation or stress at a particular material point violates the failure criterion. Tang [1] assumed that the maximum tensile strength is dominant in determining rock fracture and modeled progressive failures leading to collapse in rock and associated seismicity. van Mier et al. [9] detected local breakages by comparing the maximum allowable tensile stress with the present normal stress which was the combination of normal force and bending moment in the beam element. In the rigid-body-spring model, Bolander and Saito [16] adopted the Mohr-Coulomb criterion with a tensile cut-off, accounting for both normal and shear stresses. In the generalized beam (GB) lattice model proposed by Liu et al. [13], a failure rule similar to [16] was adopted to determine the elemental breakage, in conjunction with the consideration of separation/contact of cracked element surfaces. When a lattice serves as the micromechanics model of a material, the heterogeneous mesostructure is explicitly considered, naturally incorporating the relevant characteristic length. Thus, the explicit heterogeneity in the lattice is dominant, and the meshsize plays a secondary role when compared with homogenized macro-continuum models [18]. The crack band model proposed in [19] can be feasible and straightforward to account for such a size dependency.

In this study, we focus on the influence of ongoing local breakages to the remaining specimen. For this end, in the development of lattice-type models, there have been two main kinds of non-iterative algorithms, i.e. the load-unload method and the force-release method [17,20], which are based on two totally different assumptions in terms of characteristic time scales during progressive failures.

A post-peak softening response is generally exhibited when laboratory samples of a quasi-brittle material are loaded in tension or unconfined compression. Even though massive successful applications of lattice models in analyzing failure phenomena have been achieved, the macroscopic softening-related issue, i.e. that the load-displacement curve obtained by the lattice model shows a much lower ductility than experimental observations [20] particularly during the post-peak stage, calls for further investigations. Lilliu and van Mier [21] built a three-dimensional lattice model which was still not able to fully recur the realistic ductility. The macroscopic softening has been sometimes taken as material softening even though it is indeed a mixture of material and structural properties, resulting from the micro-cracking, rather than simply a fundamental response of the studied material [11,22-24]. van Mier [22] postulated that a lattice adopting an elastic-purely brittle fracture law at the level of the aggregates, interfaces and matrix is suitable for studying fracture mechanisms leading to global softening. Bai et al. [25], Krajcinovic and Rinaldi [26] and Li and Ren [27] emphasized that stochastic damage evolution plays an important role. Many other investigators have also proposed a lot of helpful numerical strategies for dealing with computational implementations of softening models [28,29].

The other topic to be studied is strain-rate dependency of progressive failures in quasi-brittle media, which strongly couples with the post-peak softening. For concrete, the peak strength increases and the fracture pattern becomes more scattering with increasing strain rate [30]. By proposing the extended Confinement Shear Lattice (CSL) model, Cusatis [31] investigated the effects of both the rate of crack opening and the viscoelastic deformation of the unfractured cement paste on concrete strength and fracture behavior. Wu et al. [32] made experimental observations and concluded that under intermediate strain rates, the concrete tensile strength depended on the type of tests, which cannot be captured by the Weibull effective volume method. The strain-rate sensitivity of concrete tensile failure was recurred by adopting the damaged plasticity theory combined with the strain-rate effect to describe the dynamic mechanical behavior of mortar matrix [33]. Xu and Wen [34] conducted theoretical and experimental studies on the nonlinear mechanical properties of lightweight foamed concrete under uniaxial compression over a temperature range of 223-343 K and a strain rate range of 0.001-118 per second.

In this study, both softening and strain-rate sensitivity are modeled by accounting for the viscous effect due to local breakage. It is conducted within the framework of lattice-type modeling, but the methodology can be easily extended to other computational models. We are taking the influence of ongoing local breakages on the remaining specimen as viscoelastic. When some element reaches its strength limit, its capability of bearing load vanishes suddenly and completely or significantly, leading to an immediate release of internal force in this cracking element. Such an immediate released internal force can be taken as a force suddenly applied on the remaining specimen. Therefore, the viscous effect can play an important role, i.e. the corresponding deformation of the remaining specimen somehow delays as compared with the release of internal forces of cracking elements. Based on such a physical picture, we construct a new algorithm based on a new concept, i.e. viscous force (VF) field, to deal with the above viscoelastic process. In the theoretical framework, characteristic times of both VF release and external loading are accounted for, leading to the capability of dealing with multi-time-scale problems [25].

A stepwise force-time constitutive law to be adopted here is different from the force-deformation ones (e.g., [17,28,35,36]). In literature, to keep algorithms to be sequentially linear, the original nonlinear force-deformation constitutive law is often approximated as saw-tooth shaped or piece-wise linear. The latter may be a closer approximation for an originally smooth curve. In both cases, the caused error can be overcome by adopting finer constitutive discretizations. An improved version was developed in [35]. In this study, the physical picture aforementioned, where VF evolves with time (instead of deformation), leads to a temporal constitutive law. This law is discretized stepwisely, inspired by the saw-tooth concept [28].

This paper is presented as follows. In Section 2, the GB lattice model [13,37] is briefly introduced as the mesoscale model, including a failure criterion called the modified Mohr-Coulomb rule. In Section 3, we adopt a 1D bar system to illustrate the storage and release of viscous force fields, the release of internal forces of cracking elements and their coupling mechanisms. Particularly, two kinds of stress redistributions, i.e. one due to the release of internal forces in breaking elements and the other due to the release of viscous force fields (or equivalently due to the viscous delay in the deformation of the remaining specimen), are shown in details. Subsequently, the general procedure of the proposed method, i.e. the event-driven and non- 


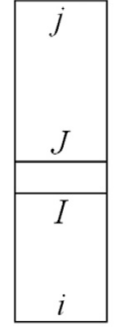

(a)

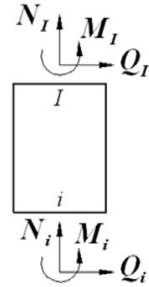

(b)

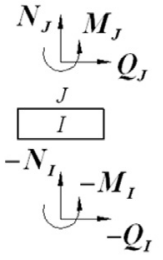

(c)

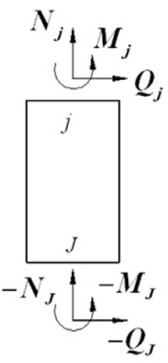

(d)

Fig. 1. Kinetics and statics of a GB lattice element.

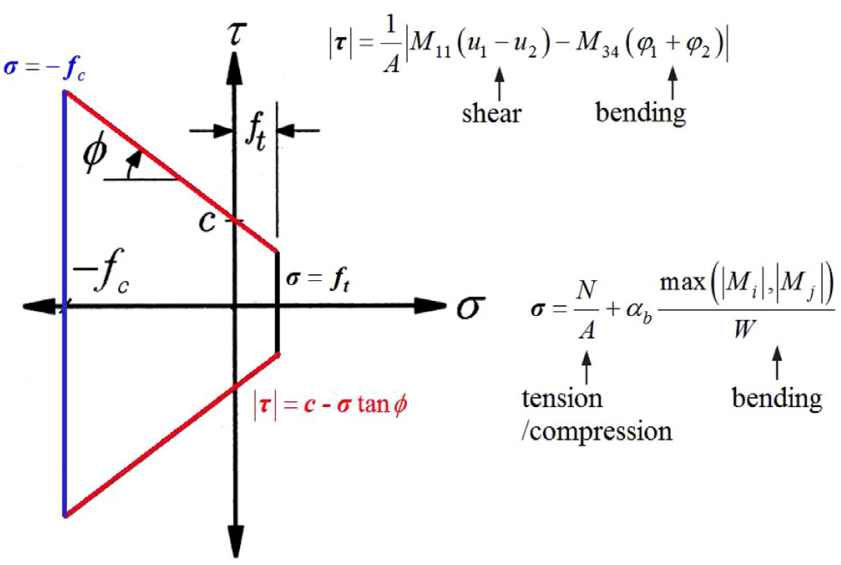

Fig. 2. Mohr-Coulomb rule with a tension and compression cut-off as the failure criterion.

iterative algorithm is proposed in Section 4. In Section 5, we conduct a series of numerical examples, showing the capability of presenting the experimentally-observed ductility (softening), more scattering fracture zones and loading rate dependence etc.

\section{GB lattice model}

The model was initially proposed by Liu et al. [13,37] for simulating the fracture process in the three-phase media like concrete. Each element can be composed of three beams with independent material properties. The response of beams can be described by the force-displacement relation of its two ends, i.e. (Fig. 1),

$$
\left\{\begin{array}{c}
Q_{1} \\
N_{1} \\
M_{1} \\
Q_{2} \\
N_{2} \\
M_{2}
\end{array}\right\}=\underbrace{\left[\begin{array}{cccccc}
M_{11} & 0 & -M_{34} & -M_{11} & 0 & -M_{34} \\
& M_{22} & 0 & 0 & -M_{22} & 0 \\
& & M_{33} & M_{34} & 0 & M_{36} \\
& & & M_{11} & 0 & M_{34} \\
& & S Y M & & M_{22} & 0 \\
& & & & & M_{33}
\end{array}\right]}_{\mathbf{M}}\left\{\begin{array}{l}
u_{1} \\
v_{1} \\
\varphi_{1} \\
u_{2} \\
v_{2} \\
\varphi_{2}
\end{array}\right\}
$$

where $\mathbf{F}_{12}=\left\{\begin{array}{llllll}Q_{1} & N_{1} & M_{1} & Q_{2} & N_{2} & M_{2}\end{array}\right\}^{T}$ and $\mathbf{u}_{12}=\left\{\begin{array}{llllll}u_{1} & v_{1} & \varphi_{1} & u_{2} & v_{2} & \varphi_{2}\end{array}\right\}^{T}$ are, respectively, the generalized force vector and the generalized displacement vector, and nonzeros $M_{i j}$ are assigned values based on the theory of the Euler-Bernoulli or Timoshenko beam. Here, in the local coordinate system on each element $i j$, the $y$-axis points from end $i$ to end $j$, and the $x$-axis is determined by rotating $y$-axis $90^{\circ}$ clockwise. The material and geometrical properties of lattice elements are calibrated based on the equivalence of strain energy [10,11,37].

Mohr-Coulomb criterion is a mathematical model describing the response of materials such as rock, rubble piles and concrete to shear stress as well as normal stress. The criterion with tension and compression cut-offs are shown in Fig. 2 and can be expressed by the following three inequations:

$$
\begin{aligned}
& \sigma<f_{t}, \\
& |\tau|<c-\sigma \tan \phi \\
& \sigma>-f_{c},
\end{aligned}
$$




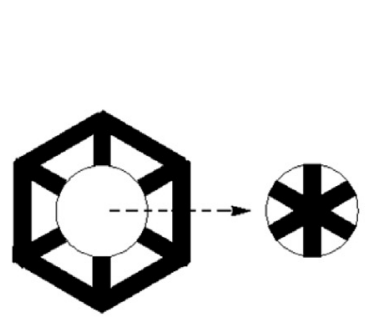

(a)
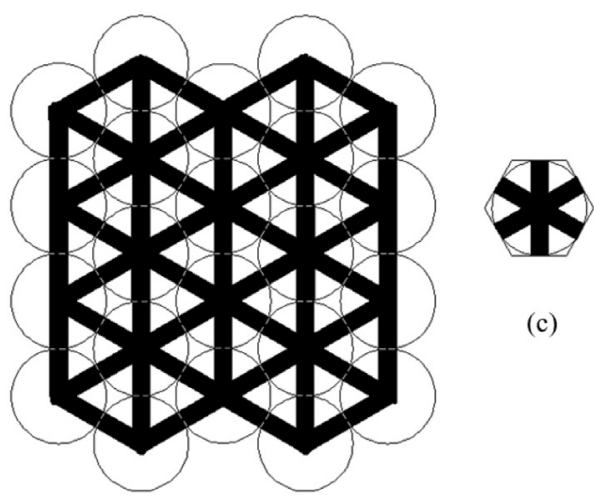

(c)

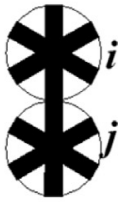

(d)

(b)

Fig. 3. Ascertainment of the contact/separation of crack surfaces.

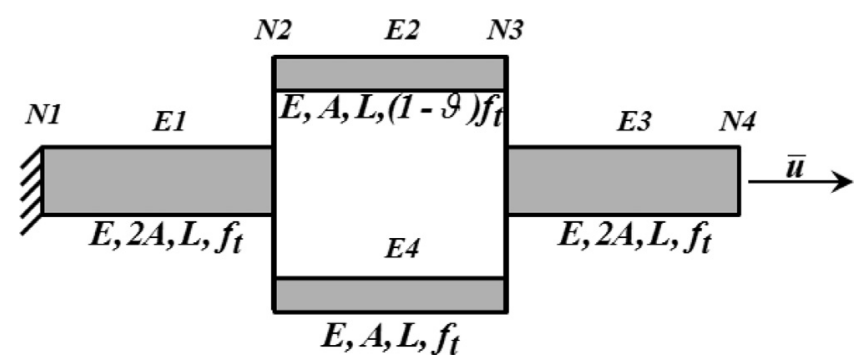

Fig. 4. A one-dimensional tensile test on a parallel-bar system including four bar element E1, E2, E3 and E4. E1's end, i.e. node N1 is fixed. E2 and E4 are installed between two vertical rigid plates which can only displace along the tension direction. A controlled displacement $\bar{u}$ is applied onto node N4 with a constant increasing rate.

where, $c$ is the cohesive strength and $\phi$ is the friction angle; $\tau$ and $\sigma$ are the shear stress and the normal stress, respectively; $f_{t}$ and $f_{c}$ are the tensile and compressive strengths, respectively. The normal stress can be written as

$$
\sigma=\frac{N}{A}+\alpha_{b} \frac{\max \left(\left|M_{i}\right|,\left|M_{j}\right|\right)}{W},
$$

where, $N$ is the normal force, $M_{i}$ and $M_{j}$ are the bending moments at node $i$ and $j$, respectively, in the beam studied, $W$ is the section modulus, the coefficient $\alpha_{b}$ regulates what part of the bending moment is considered. While the shear stress can be calculated as

$$
|\tau|=\frac{1}{A}\left|M_{11}\left(u_{1}-u_{2}\right)-M_{34}\left(\varphi_{1}+\varphi_{2}\right)\right|
$$

with $A$ the area of the cross-section.

Fracture surfaces produced previously can come into contact with each other under a varying loading, therefore it is required to take into account fracture surface contacts. For this end, the material domain around every node is assumed to be circular, as shown in Fig. 3. Thus contacts can be ascertained by checking the present distance between centers of two neighboring round disks.

\section{Storage and release of viscous force fields: an illustrative 1D example}

Consider a one-dimensional parallel bar system which is composed of four link elements, i.e. E1, E2, E3 and E4, as shown in Fig. 4. All elements have a common length $L$ and Young's modulus $E$. The cross-section of E1 and E3 is $2 A$, while that of E2 and E4 is $A$. The material is assumed to be elastoviscous. The structure is under a gradually increased tension. In the present work, we assume that the external loading rate is so slow that both the viscosity due to external loading and the inertia effect can be neglected. E1, E3 and E4 have a common tensile strength, i.e. $f_{t}$, while that of E2 is equal to $(1-\vartheta) f_{t}$, where $0<\vartheta<1$.

Among these four elements, E2 is the first to reach its breakage limit. At this critical time, which is noted as $t_{0}^{-}$, the controlled displacement is given as

$$
\bar{u}_{0}=\frac{3(1-\vartheta) L f_{t}}{E}
$$




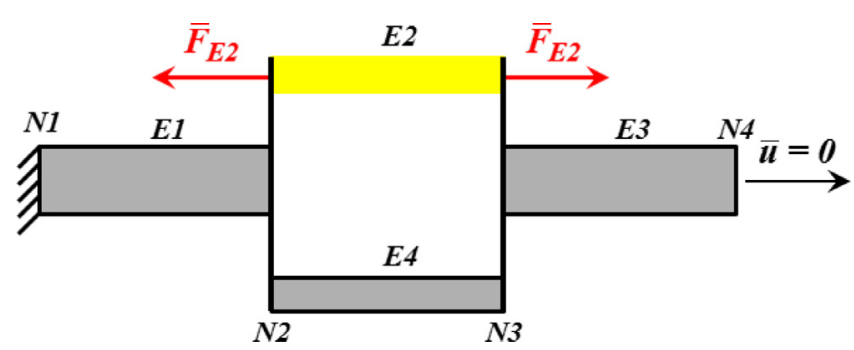

Fig. 5. Boundary and loading conditions for calculating the trial incremental displacement field $\Delta \boldsymbol{D}_{\mathbf{1}}\left(t_{0}\right)$.

the total displacement field is written as

$$
\boldsymbol{D}\left(t_{0}^{-}\right)=\bar{u}_{0}\left\{\begin{array}{llll}
0 & \frac{1}{3} & \frac{2}{3} & 1
\end{array}\right\},
$$

and the internal force of $\mathrm{E} 2$ is

$$
\bar{F}_{\mathrm{E} 2}=\frac{E A \bar{u}_{0}}{3 L} .
$$

The internal force field, which is composed of internal forces of all elements, can be expressed in the form,

$$
\mathbf{F}\left(t_{0}^{-}\right)=\bar{F}_{\mathrm{E} 2}\left\{\begin{array}{llll}
2 & 1 & 2 & 1
\end{array}\right\},
$$

where the sign of tensile and compressive internal force is taken as positive and negative, respectively. Or equivalently, a positive and negative internal axial force is like " $\leftarrow \rightarrow$ " and " $\rightarrow \leftarrow$ ", respectively.

Immediately after the above critical time, i.e. $t_{0}^{+}$, E2's capacity of bearing load totally vanishes, which leads to the release of its internal force into the other elements, i.e. E1, E3 and E4. At the moment $t_{0}^{-}$when E2 becomes critical but keeps intact, its contribution to the remaining specimen is a force with the magnitude $\bar{F}_{\mathrm{E} 2}$ applied at its two ends, N2 and N3, in opposite directions, i.e. " $\rightarrow \leftarrow$ ", indicating that the force direction at N2 is $\rightarrow$ and that at N3 is $\leftarrow$. Apparently, if E2 is eliminated from the system while keeping the $\bar{F}_{\mathrm{E} 2}(\rightarrow \leftarrow)$ at its ends, the displacement field of the remaining specimen will be the same as that at $t_{0}^{-}$, i.e. $\boldsymbol{D}\left(t_{0}^{-}\right)$. Thus, to consider the influence of E2's breakage on the rest, two forces, which are equal to $\overline{\mathrm{F}}_{\mathrm{E} 2}$, are respectively applied onto the two ends of E2, along the direction like " $\leftarrow \rightarrow$ ", intending to contradict the action of previous intact E2. As shown in Fig. 5, under the condition that the controlled displacement is set to zero, i.e. $\bar{u}=0$, and $\bar{F}_{\mathrm{E} 2}$ is fully released at E2's two ends, we can obtain a trial incremental displacement field,

$$
\triangle \boldsymbol{D}_{\mathbf{1}}\left(t_{0}\right)=\frac{\bar{u}_{0}}{12}\left\{\begin{array}{llll}
0 & -1 & 1 & 0
\end{array}\right\}
$$

Notably, $\Delta \boldsymbol{D}_{\mathbf{1}}\left(t_{0}\right)$ is simply trial, and it can become real by multiplying a coefficient $\lambda$ to it, which will be discussed below. Thus, no operation of judging new breakage(s) is required in $\boldsymbol{D}\left(t_{0}^{-}\right)+\Delta \boldsymbol{D}_{\mathbf{1}}\left(t_{0}\right)$. So far, the operation of stress redistribution is the same as that discussed in [20].

The above releasing of E2's internal forces is assumed to happen during an infinitesimal time, i.e. from $t_{0}^{-}$to $t_{0}^{+}$, when compared with the characteristic time scale of external loading. Therefore, simultaneously, the remaining structure composed of E1, E3 and E4 is deemed to experience a suddenly-applied loading, and thus viscosity cannot be neglected. In other words, deformations in E1, E3 and E4 need to take some time to become fully consistent with the updated loading conditions (the internal forces to be released is taken as external loading). Note that for the calculation of the trial displacement field $\Delta \boldsymbol{D}_{\mathbf{1}}\left(t_{0}\right)$, the specimen is temporarily assumed to be purely elastic with no viscosity. While the viscous effect will be recurred by the imposition of a viscous force field, which will be demonstrated below.

During the period from $t_{0}^{-}$to $t_{0}^{+}$, E2's internal force to be released increases from zero to $\bar{F}_{\mathrm{E} 2}$; but the simultaneous change of length in E1, E3 and E4 will be smaller than the counterpart when no viscous effect is considered. To describe the extend the material can deform immediately corresponding to the releasing of internal force of the breaking element, a parameter $\alpha(0 \leq \alpha \leq 1)$, which is deemed to be material-dependent, is introduced to represent the relative share of immediate elasticity. Then, when $\lambda \bar{F}_{\mathrm{E} 2}(0 \leq \lambda<1)$ has been released since the moment $t_{0}^{-}$and $\mathrm{E} 4$ has not reached its strength limit, the immediate change of length in E1 and E3 is $-\alpha \lambda \bar{u}_{0} / 12$, and that in E4 is $\alpha \lambda \bar{u}_{0} / 6$.

To implement the above-mentioned deformation-delaying phenomenon, we propose a new method whereby a proper kind of viscous force fields is firstly constructed and then gradually released. In preparation for the derivation in Sections 3.1 and 3.2, we compute the trial force field, $\Delta \mathbf{V}_{\mathbf{1}}\left(t_{0}\right)$, arising in the trial incremental displacement field $-\Delta \boldsymbol{D}_{\mathbf{1}}\left(t_{0}\right)$, which can be expressed as,

$$
\triangle \mathbf{V}_{1}\left(t_{0}\right)=\frac{1}{2} \bar{F}_{\mathrm{E} 2}\left\{\begin{array}{llll}
1 & 0 & 1 & -1
\end{array}\right\}
$$

where the sign of internal force is assigned in the same way as in Eq. (3.4). 


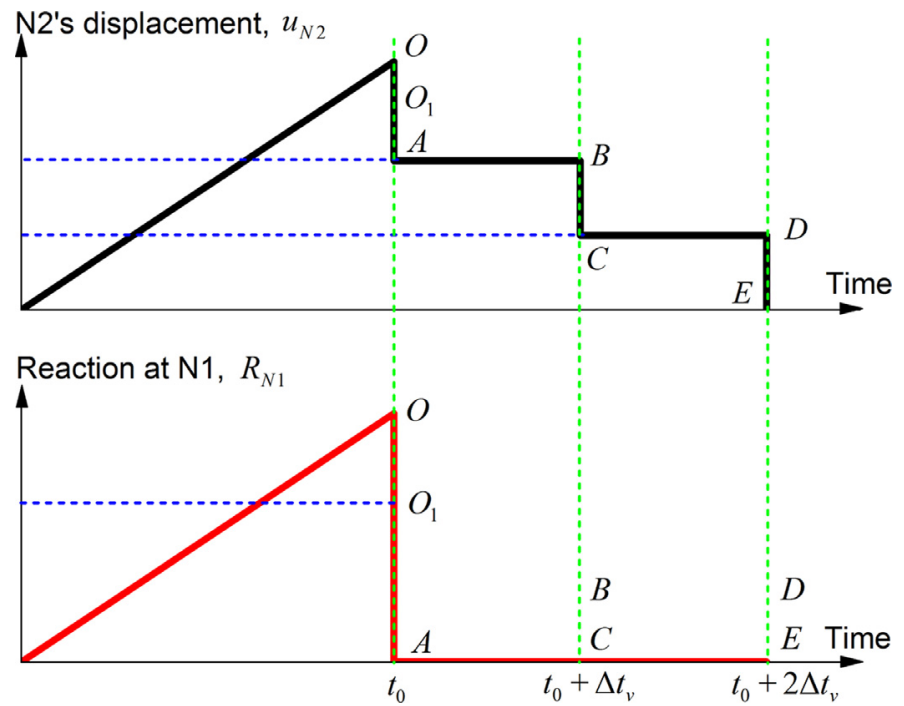

Fig. 6. In case 1 , the curves of the reaction force at $\mathrm{N} 1, R_{\mathrm{N} 1}$, and the displacement of $\mathrm{N} 2$ versus time.
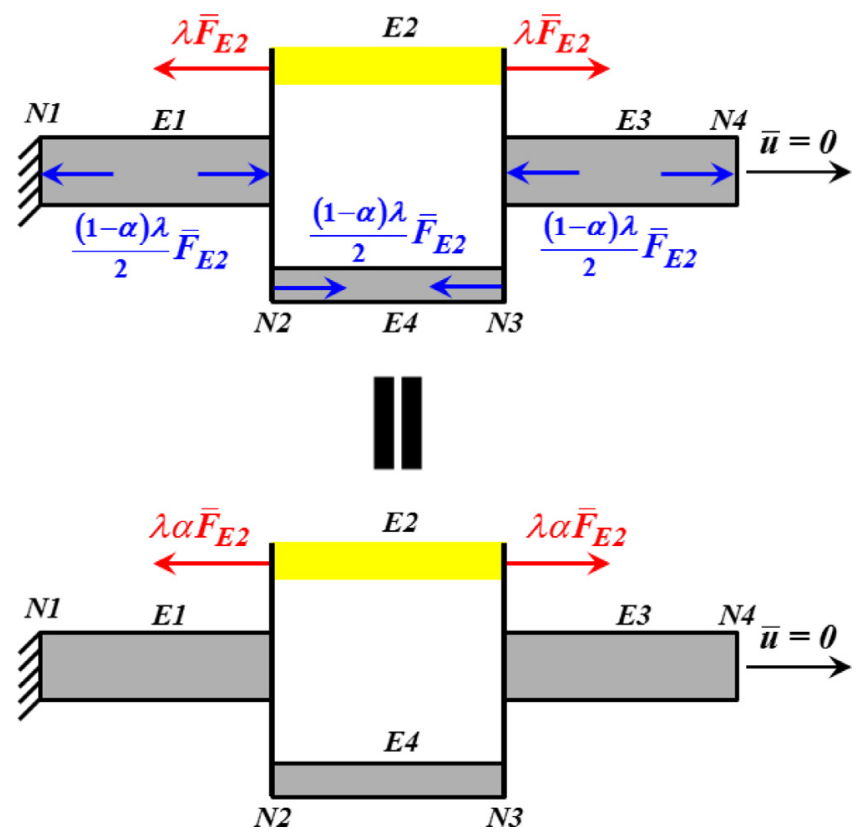

Fig. 7. Boundary and loading conditions for calculating the incremental displacement field bridging $O$ and $O_{1}, \alpha \lambda \triangle \boldsymbol{D}_{\mathbf{1}}\left(t_{0}\right)$.

In this study, we assume that the viscosity is purely due to the occurrence of breakages, hence, there is no viscous force at the time $t_{0}^{-}$, i.e.,

$$
\mathbf{v}\left(t_{0}^{-}\right)=\left\{\begin{array}{llll}
0 & 0 & 0 & 0
\end{array}\right\} .
$$

There are two possible cases for consideration: Case 1 where element E4 is induced to break due to the release of E2's internal force under the fixed controlled displacement, $\bar{u}_{0}$, and Case 2 where E4 keeps intact when E2's internal force has been fully released under $\bar{u}_{0}$.

\subsection{Case 1: when E4 is induced to break by releasing E2's internal forces}

As shown in Fig. 6, due to the release of E2's internal force, both the reaction force at node N1, $R$, and the displacement at node $\mathrm{N} 2, u_{\mathrm{N} 2}$, decrease under the fixed external controlled displacement $\bar{u}_{0}$. Assume that when $R$ drops to point $O_{1}$, E4 reaches a critical state. 


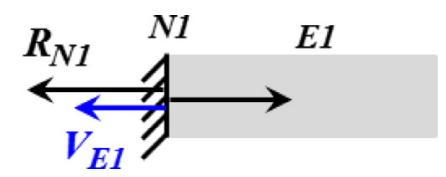

Fig. 8. Calculation of the reaction force at N1, $R_{\mathrm{N} 1}$ at the time of $\lambda=\lambda_{1}$, which corresponds to the point $O_{1}$ in Fig. 6 .

\subsection{1. $\mathrm{O} \rightarrow \mathrm{O}_{1}$}

The incremental displacement field is calculated for the specimen with E2 eliminated under the following boundary and loading conditions (Fig. 7): nodes N1 and N4 are fixed, part of E2's internal force, i.e. $\lambda \bar{F}_{\mathrm{E} 2}$, is released at nodes N2 and N3, and a viscous force field, $(1-\alpha) \lambda \triangle \mathbf{V}_{1}\left(t_{0}\right)$, is imposed on the specimen. We assume that when $\lambda=\bar{\lambda}_{1}$, E4 becomes critical. When the reaction force $R$ drops from point $O$ to $O_{1}, \lambda$ increases from 0 to $\bar{\lambda}_{1}$. Thus, the incremental displacement field obtained is $\alpha \lambda \triangle \boldsymbol{D}_{\mathbf{1}}\left(t_{0}\right)$, which can also be calculated under the equivalent conditions: N1 and N4 are fixed, and part of E2's internal force, $\alpha \lambda \bar{F}_{\mathrm{E} 2}$, which is corresponding to immediate elasticity, is released at nodes N2 and N3. When $\lambda=\bar{\lambda}_{1}$, E4's strain is given by

$$
\frac{f_{t}}{E}=(1-\vartheta) \frac{f_{t}}{E}+\alpha \bar{\lambda}_{1} \frac{(1-\vartheta) f_{t}}{2 E}
$$

where $\bar{\lambda}_{1}=2 \vartheta / \alpha(1-\vartheta)$. Apparently, $\bar{\lambda}_{1}>1$ indicates that E4 will not be induced to break by the release of E2's internal force, which will be discussed in Section 3.2. Practically, the critical value cannot be bigger than 1, i.e.,

$$
\lambda_{1}=\min \left(1, \bar{\lambda}_{1}\right)
$$

and the corresponding internal force in E4 is given by $\bar{F}_{\mathrm{E} 4}=\frac{E A}{L} \bar{u}_{0}\left(\frac{1}{3}+\frac{\alpha \lambda_{1}}{6}\right)$.

In the calculation carried out in Fig. 7 , since the viscosity is represented by the VF field, $(1-\alpha) \lambda \Delta \mathbf{V}_{1}\left(t_{0}\right)$, the material is still set to be immediately elastic. In this sense, the VF concept helps to avoid the needs of developing non-elastic constitutive models.

In summary, at point $O_{1}$, i.e., at the time when $\lambda=\lambda_{1}$, there are

- The displacement field:

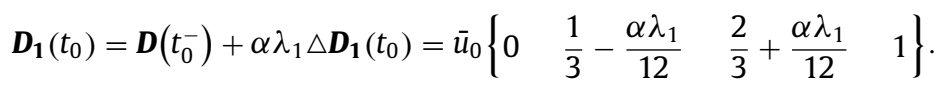

- The VF field stored during the period whereby $\lambda$ changes from 0 to $\lambda_{1}$ :

$$
\mathbf{V}_{1}\left(t_{0}\right)=\mathbf{v}\left(t_{0}^{-}\right)+\Delta \mathbf{V}_{1}\left(t_{0}\right)=\frac{1-\alpha}{2} \lambda_{1} \bar{F}_{\mathrm{E} 2}\left\{\begin{array}{llll}
1 & 0 & 1 & 0
\end{array}\right\}
$$

- The internal force field which is calculated based on $\boldsymbol{D}_{\mathbf{1}}\left(t_{0}\right)$ :

$$
\mathbf{F}_{1}\left(t_{0}\right)=\frac{2 E A \bar{u}_{0}}{3 L}\left(1-\frac{\alpha \lambda_{1}}{4}\right)\left\{\begin{array}{llll}
1 & 0 & 1 & 0
\end{array}\right\} .
$$

- E2's internal force to be released at nodes N2 and N3: $\left(1-\lambda_{1}\right) \bar{F}_{\mathrm{E} 2}$.

- E4's internal force to be released at nodes N2 and N3: $\bar{F}_{\mathrm{E} 4}=\frac{E A}{L} \bar{u}_{0}\left(\frac{1}{3}+\frac{\alpha \lambda_{1}}{6}\right)$.

- The VF having been stored in E4 during $O \rightarrow O_{1}$, needs to be released at N2 and N3 after $O_{1}$ :

$$
\frac{1-\alpha}{2} \lambda_{1} \bar{F}_{\mathrm{E} 2}(\leftarrow \rightarrow)
$$

Since E4 will be eliminated, so the VF stored in it so far, i.e. $\frac{1-\alpha}{2} \lambda_{1} \bar{F}_{\mathrm{E} 2}$, as shown in Eq. (3.11), would be released in the same manner as the cracking element's internal force.

- Reaction force at node $\mathrm{N} 1$ (Fig. 8): $R_{\mathrm{N} 1}=F_{\mathrm{E} 1}-V_{\mathrm{E} 1}=\frac{E A \bar{u}_{0}}{6 L}\left(4-\lambda_{1}\right)$.

It is notable that the introduction of the above VF field satisfies the equilibrium condition. For example, let us consider node N2, as shown in Fig. 9. At the time of $\lambda=\lambda_{1}$, internal force of E1 is $F_{\mathrm{E} 1}=\frac{2 E A \bar{u}_{0}}{3 L}\left(1-\frac{\alpha \lambda_{1}}{4}\right)$, the VFs in E1 and $\mathrm{E} 4$ are $V_{\mathrm{E} 1}=\frac{\lambda_{1} E A \bar{u}_{0}}{6 L}(1-\alpha)$ and $V_{\mathrm{E} 4}=-\frac{\lambda_{1} E A \bar{u}_{0}}{6 L}(1-\alpha)$ respectively; internal forces of E2 and E4 waiting to be released are, respectively, $\left(1-\lambda_{1}\right) \bar{F}_{\mathrm{E} 2}$ and $\bar{F}_{\mathrm{E} 4}$. All above forces at $\mathrm{N} 2$ satisfy the equilibrium condition, i.e.

$$
\bar{F}_{\mathrm{E} 4}+V_{\mathrm{E} 1}+V_{\mathrm{E} 4}+\left(1-\lambda_{1}\right) \bar{F}_{\mathrm{E} 2}-F_{\mathrm{E} 1}=0 .
$$




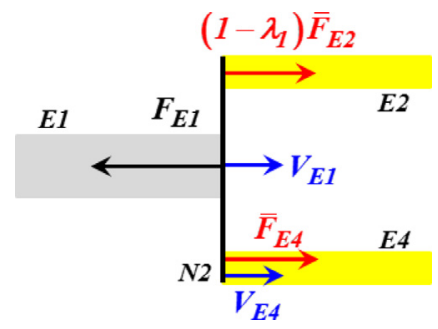

Fig. 9. Equilibrium condition at N2, at the time of $\lambda=\lambda_{1}$, which is corresponding to the point $O_{1}$ in Fig. 6 .

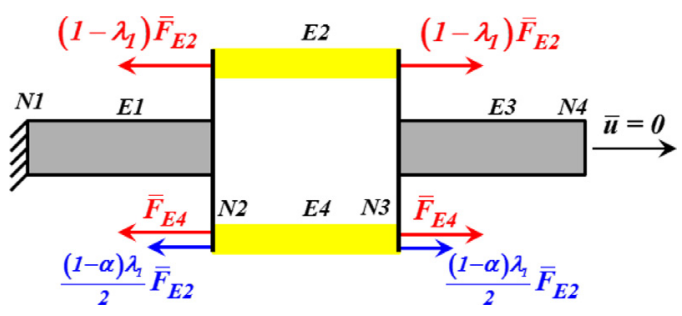

Fig. 10. In case 1 , boundary and loading conditions for calculating the trial incremental displacement field, $\Delta \boldsymbol{D}_{2}\left(t_{0}\right)$, which bridges $O_{1}$ and $A$ in Fig. 6 .

\subsection{2. $\mathrm{O}_{1} \rightarrow \mathrm{A}$ process}

Since no further elemental breakage can possibly happen at the present level of controlled displacement, i.e. $\bar{u}_{0}$, the period from the time of E4 becoming critical to point A, as shown in Fig. 6, i.e. the time when all internal forces in cracking elements, E2 and E4, have been released, can be taken as one single linearly elastic process. Again, we firstly construct the trial incremental displacement field $\triangle \boldsymbol{D}_{\mathbf{2}}\left(t_{0}\right)$, which arises under the boundary and loading conditions, as shown in Fig. 10: in the specimen where E2 and E4 are eliminated, nodes N1 and N4 are fixed, we simultaneously release the remaining internal force of E2, $\left(1-\lambda_{1}\right) \bar{F}_{\mathrm{E} 2}$, the internal force of E4, $\bar{F}_{\mathrm{E} 4}$, and the viscous force stored in E4, which needs to be released due to E4's breakage, i.e. $\frac{1-\alpha}{2} \lambda_{1} \bar{F}_{\mathrm{E} 2}$. The produced displacement field is

$$
\triangle \boldsymbol{D}_{2}\left(t_{0}\right)=\frac{\left(4-\lambda_{1}\right) \bar{u}_{0}}{12}\left\{\begin{array}{llll}
0 & -1 & 1 & 0
\end{array}\right\} .
$$

Because there is no further induced breakage, the scaling factor for $\Delta \boldsymbol{D}_{2}\left(t_{0}\right)$ is $\lambda_{2}=1$, the real incremental displacement is equal to $\alpha \Delta \boldsymbol{D}_{\mathbf{2}}\left(t_{0}\right)$. Correspondingly, the incremental VF field should be calculated in the displacement field $-(1-\alpha) \triangle \boldsymbol{D}_{\mathbf{2}}\left(t_{0}\right)$, i.e.,

$$
\triangle \mathbf{V}_{2}\left(t_{0}\right)=\frac{E A \bar{u}_{0}}{6 L}\left(4-\lambda_{1}\right)(1-\alpha)\left\{\begin{array}{llll}
1 & 0 & 1 & 0
\end{array}\right\}
$$

At the time $t_{0}^{+}$, which is corresponding to point A in Fig. 6, there are,

- The displacement field:

$$
\begin{aligned}
\boldsymbol{D}\left(t_{0}^{+}\right) & =\boldsymbol{D}\left(t_{0}^{-}\right)+\alpha \lambda_{1} \triangle \boldsymbol{D}_{1}\left(t_{0}\right)+\alpha \Delta \boldsymbol{D}_{2}\left(t_{0}\right) \\
& =\bar{u}_{0}\left\{\begin{array}{llll}
0 & \frac{1-\alpha}{3} & \frac{2+\alpha}{3} & 1
\end{array}\right\} .
\end{aligned}
$$

- The internal force field:

$$
\mathbf{F}\left(t_{0}^{+}\right)=\frac{2 E A \bar{u}_{0}(1-\alpha)}{3 L}\left\{\begin{array}{llll}
1 & 0 & 1 & 0
\end{array}\right\}
$$

which is calculated based on the displacement field $\boldsymbol{D}\left(t_{0}^{+}\right)$given in Eq. (3.17).

- The VF field stored so far:

$$
\begin{aligned}
\mathbf{v}\left(t_{0}^{+}\right) & =\mathbf{V}_{\mathbf{1}}\left(t_{0}\right)+\Delta \mathbf{v}_{2}\left(t_{0}\right) \\
& =\frac{2 E A \bar{u}_{0}(1-\alpha)}{3 L}\left\{\begin{array}{llll}
1 & 0 & 1 & 0
\end{array}\right\}
\end{aligned}
$$

where, $\mathbf{v}_{\mathbf{1}}\left(t_{0}\right)$ is given in Eq. (3.11).

- E2's internal force to be released at nodes N2 and N3: 0 .

- E4's internal force to be released at nodes N2 and N3: 0.

- Reaction force at node N1:

$$
R_{\mathrm{N} 1}=F_{\mathrm{E} 1}-V_{\mathrm{E} 1}=0 .
$$

It is a well-recognized fact that no matter how viscosity plays its role, the reaction force should become zero after the specimen is completely broken into two. 


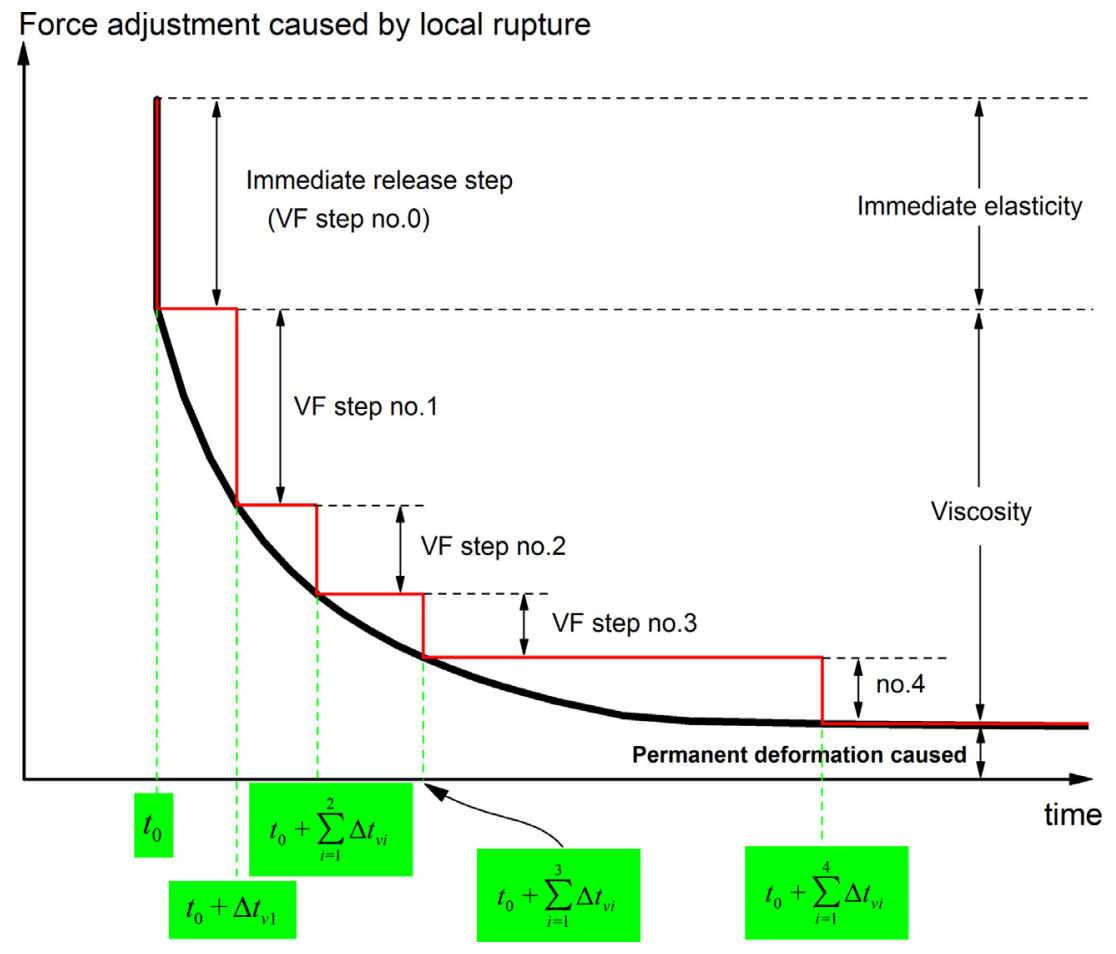

Fig. 11. The viscoelastic response caused by local rupture, and its stepwise approximation. Here the total of VF steps is taken as $N=4$.

\subsection{3. $\mathrm{A} \rightarrow \mathrm{B} \rightarrow \mathrm{C} \rightarrow \mathrm{D} \rightarrow \mathrm{E}$ : stepwise release of the $V \mathrm{~F}$ field}

From $O$ to $A$, the release of the internal forces in critical elements causes two consequences: one is the immediate elastic response, i.e. $\boldsymbol{D}\left(t_{0}^{+}\right)-\boldsymbol{D}\left(t_{0}^{-}\right)$, the other is the storage of the VF field, $\mathbf{V}\left(t_{0}^{+}\right)$, which is corresponding to a delay in deformation due to viscosity.

Immediately after time $t_{0}^{+}$, the VF field would gradually decay to zero, corresponding to the gradual vanishing of the deformation delay, which is numerically implemented in a stepwise manner, as shown in Fig. 11. The VF field stored in the remaining specimen would be released to zero by taking $N$ equal or unequal steps. We denote the $i$ th decreasing VF step as the $i$ th VF step. The proportion of the released VF field in each VF step is calculated according to the viscous properties of the material studied. Particularly, during the stress redistributing process due to the occurrence of new breakages, the specimen will simultaneously and partially adjust the deformation behavior, which is called immediate elasticity. For consistency, such an immediate stress adjustment is taken as the 0th VF step.

Notably, Fig. 11 also indicates a temporal energy dissipation associated with local rupture. In previous non-iterative sequentially linear algorithms like the F-R method (e.g., [17,20]), the material is assumed to be purely immediately elastic (without any viscosity), so the local rupture-induced energy dissipation rate is infinite. Nevertheless, in Fig. 11, the whole energy to be dissipated includes an immediately-elastic and viscous part; the former dissipates infinitely fast during the 0th step, and the latter does in a finite rate under the drive of the gradual VF release during the 1-N th steps.

Before proceeding with future works, we need to adopt a more informative notation for the $\mathrm{VF}$ field $\mathbf{V}\left(t_{0}^{+}\right)$, i.e.,

$$
\mathbf{v}\left(t_{0}^{+}\right)=\mathbf{v}\left(t_{0}, \quad n_{v}, \quad \phi_{t}, \quad \phi_{v}, \quad \mathbb{R}\right),
$$

where, in the parameter list on the right side of the equation, $t_{0}$ means the time at which the VF field is stored; $n_{v} \in$ $\{1,2, \ldots, N\}$ is the number of the present VF step; $\phi_{t}$ is used to note how much time has elapsed among the whole step time span $\Delta t_{n_{v}}$, thus the present time is $t=t_{0}+\sum_{i=1}^{n_{v}-1} \Delta t_{i}+\phi_{t} \Delta t_{n_{v}} ; \phi_{v}$ means that in the present VF step, an incremental VF field $\phi_{v} \Delta \mathbf{V}_{\mathbf{n}_{\mathbf{v}}}$ has been released, where $\Delta \mathbf{V}_{\mathbf{n}_{\mathrm{v}}}$ is the total VF that should be released during the present VF step; $\mathbb{R}$ is a set that includes all ruptures arisen from $t_{0}^{+}$to the present time, and therefore it is a null set, i.e. $\emptyset$, at the time $t_{0}^{+}$.

In the 1D example studied, for the sake of simplicity and without loss of generality, we assume that the VF field stored from $t_{0}^{-}$to $t_{0}^{+}$takes $N=2$ equal VF steps to decrease to zero, and the common time duration of each VF step is written as $\Delta t_{v}\left(=\Delta t_{1}=\Delta t_{2}\right)$, and $(1-\alpha) \mathbf{v}\left(t_{0}^{+}\right) / n_{v}$ is released into the remaining specimen in each VF step.

$A \rightarrow B$ process:

The increment in the controlled displacement at node $N 4$ is $\frac{\Delta t_{v}}{t_{0}} \bar{u}_{0}$. At point $B$, i.e. the time $\left(t_{0}+\Delta t_{v}\right)^{-}$, there are, 
- The displacement field:

$$
D\left(\left(t_{0}+\Delta t_{v}\right)^{-}\right)=\bar{u}_{0}\left\{\begin{array}{llll}
0 & \frac{1-\alpha}{3} & \frac{2+\alpha}{3}+\frac{\Delta t_{\nu}}{t_{0}} & 1+\frac{\Delta t_{v}}{t_{0}}
\end{array}\right\} .
$$

- The VF field is kept unchanged:

$$
\mathbf{v}\left(t_{0}, 1, \quad 1, \quad 0, \quad \emptyset\right)=\mathbf{v}\left(t_{0}^{+}\right)=\frac{2 E A \bar{u}_{0}(1-\alpha)}{3 L}\left\{\begin{array}{llll}
1 & 0 & 1 & 0
\end{array}\right\} .
$$

- The internal force field is kept unchanged:

$$
\mathbf{F}\left(\left(t_{0}+\Delta t_{v}\right)^{-}\right)=\mathbf{F}\left(t_{0}^{+}\right)=\frac{2 E A \bar{u}_{0}(1-\alpha)}{3 L}\left\{\begin{array}{llll}
1 & 0 & 1 & 0
\end{array}\right\} .
$$

$B \rightarrow C$ process:

The real incremental displacement field is calculated under the following condition: In the specimen with E2 and E4 eliminated, the controlled displacement at node $\mathrm{N} 4$ is zero, and a force field equal to $-\frac{1}{2} \mathbf{v}\left(t_{0}, 1, \quad 1, \quad 0, \quad \emptyset\right)$ is applied. At point $C$, i.e. the time $\left(t_{0}+\Delta t_{v}\right)^{+}$, there are,

- The displacement field:

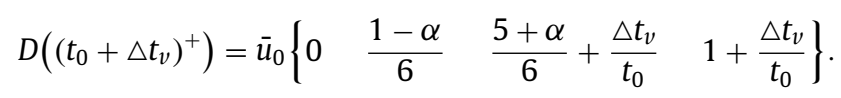

- The VF field:

$$
\mathbf{v}\left(t_{0}, 2, \quad 0, \quad 0, \quad \emptyset\right)=\frac{E A \bar{u}_{0}(1-\alpha)}{3 L}\left\{\begin{array}{llll}
1 & 0 & 1 & 0
\end{array}\right\} .
$$

- The internal force field:

$$
\mathbf{F}\left(\left(t_{0}+\Delta t_{v}\right)^{+}\right)=\frac{E A \bar{u}_{0}(1-\alpha)}{3 L}\left\{\begin{array}{llll}
1 & 0 & 1 & 0
\end{array}\right\} .
$$

$C \rightarrow$ D process:

The increment in the controlled displacement at node N4 is $\frac{\Delta t_{v}}{t_{0}} \bar{u}_{0}$. At point $D$, i.e. the time $\left(t_{0}+2 \Delta t_{v}\right)^{-}$, there are,

- The displacement field:

$$
D\left(\left(t_{0}+2 \triangle t_{v}\right)^{-}\right)=\bar{u}_{0}\left\{\begin{array}{llll}
0 & \frac{1-\alpha}{6} \quad \frac{5+\alpha}{6}+2 \frac{\Delta t_{v}}{t_{0}} & 1+2 \frac{\Delta t_{v}}{t_{0}}
\end{array}\right\} .
$$

- The VF field:

$$
\mathbf{v}\left(t_{0}, 2, \quad 1, \quad 0, \quad \emptyset\right)=\frac{E A \bar{u}_{0}(1-\alpha)}{3 L}\left\{\begin{array}{llll}
1 & 0 & 1 & 0
\end{array}\right\} .
$$

- The internal force field:

$$
\mathbf{F}\left(\left(t_{0}+2 \triangle t_{v}\right)^{+}\right)=\frac{E A \bar{u}_{0}(1-\alpha)}{3 L}\left\{\begin{array}{llll}
1 & 0 & 1 & 0
\end{array}\right\} .
$$

$D \rightarrow E$ process:

The real incremental displacement field is calculated under the following condition: In the specimen with E2 and E4 eliminated, the controlled displacement at node $\mathrm{N} 4$ is zero, and a force field equal to $-\mathrm{V}\left(t_{0}, 2, \quad 1, \quad 0, \quad \emptyset\right)=$ $-\frac{1}{2} \mathbf{V}\left(t_{0}, 1, \quad 1, \quad 0, \quad \emptyset\right)$ is applied. At point $E$, i.e. the time $\left(t_{0}+2 \Delta t_{v}\right)^{+}$, there are,

- The displacement field:

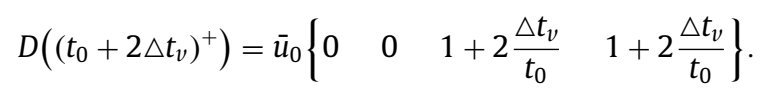

- The VF field:

$$
\mathbf{v}\left(t_{0}, \quad 2, \quad 1, \quad 1, \quad \emptyset\right)=\left\{\begin{array}{llll}
0 & 0 & 0 & 0
\end{array}\right\} .
$$

- The internal force field:

$$
\mathbf{F}\left(\left(t_{0}+2 \triangle t_{v}\right)^{+}\right)=\left\{\begin{array}{llll}
0 & 0 & 0 & 0
\end{array}\right\} .
$$




\subsection{Case 2: when $\mathrm{E} 4$ is induced to break by the VF release}

As for the example given in Section 3.1, it is actually a pure VF releasing process after the time $t_{0}^{+}$(point A in Fig. 6), which is suitable to show the proposed algorithm to store up and release VF fields in a separate manner.

Nevertheless, in real failures, relaxations of VF fields and appearances of new breakages are often coupled with and influenced by each other. To illustrate such a coupling mechanism, the following case is presented: in the specimen, E2 breaks at $t_{0}$, and E4 breaks at $t_{0}+\Delta t_{v}$. The corresponding curves are shown in Fig. 12 The key states during the whole failure process are tracked one by one in the following.

Point 0:

- The displacement field:

$$
D\left(t_{0}^{-}\right)=\bar{u}_{0}\left\{\begin{array}{llll}
0 & \frac{1}{3} & \frac{2}{3} & 1
\end{array}\right\}
$$

where, $\bar{u}_{0}=\frac{3 L f_{t}(1-\theta)}{E}$.

- There is no VF field so far.

- The internal force field:

$$
\mathbf{F}\left(t_{0}^{-}\right)=\frac{2 E A \bar{u}_{0}}{3 L}\left\{\begin{array}{llll}
1 & 0 & 1 & \frac{1}{2}
\end{array}\right\} .
$$

- E2's internal force to be released at nodes $\mathrm{N} 2$ and N3: $\bar{F}_{\mathrm{E} 2}=\frac{E A \bar{u}_{0}}{3 L}$.

- Reaction force at $\mathrm{N} 1: R\left(t_{0}^{-}\right)=\frac{2 E A \bar{u}_{0}}{3 L}$.

$O \rightarrow$ A process:

The trial incremental displacement field is calculated under the following conditions (which are the same as those for calculating $\triangle \boldsymbol{D}_{\mathbf{1}}\left(t_{0}\right)$ in Section 3.1.2, as shown in Fig. 5): in the specimen with E2 eliminated, the controlled displacement at node N4 is zero, and E2's internal force, i.e. $\bar{F}_{\mathrm{E} 2}$, is released at nodes N2 and N3, which is accompanied by the storage of corresponding incremental VF field. At point $A$, i.e. the time $t_{0}^{+}$, there are,

- The displacement field:

$$
D\left(t_{0}^{+}\right)=\bar{u}_{0}\left\{0 \quad \frac{1}{3}-\frac{\alpha}{12} \quad \frac{2}{3}+\frac{\alpha}{12} \quad 1\right\} .
$$

- The viscous force field:

$$
\mathbf{v}\left(t_{0}, \quad 1, \quad 0, \quad 0, \quad \emptyset\right)=\frac{(1-\alpha) E A \bar{u}_{0}}{6 L}\left\{\begin{array}{llll}
1 & 0 & 1 & -1
\end{array}\right\} .
$$

We note

$$
\overline{\mathbf{v}}\left(t_{0}\right)=\mathbf{v}\left(t_{0}, \quad 1, \quad 0, \quad 0, \quad \emptyset\right),
$$

and therefore, VF stored in element $i$ is written as $\bar{V}_{E i}\left(t_{0}\right)$. For example, $\bar{V}_{\mathrm{E} 4}\left(t_{0}\right)=-\frac{(1-\alpha) E A \bar{u}_{0}}{6 L}$.

- The internal force field:

$$
\mathbf{F}\left(t_{0}^{+}\right)=\frac{2 E A \bar{u}_{0}}{3 L}\left\{\left(1-\frac{\alpha}{4}\right) \quad 0 \quad\left(1-\frac{\alpha}{4}\right) \quad \frac{1}{2}\left(1+\frac{\alpha}{2}\right)\right\} .
$$

- There is no internal force left to be released.

- Reaction force at $\mathrm{N} 1: R\left(t_{0}^{+}\right)=\frac{E A \bar{u}_{0}}{3 L}$.

$A \rightarrow B$ process:

The incremental displacement field is calculated under the following condition: in the specimen with E2 eliminated, the controlled displacement at node N4 is $\frac{\Delta t_{v}}{t_{0}} \bar{u}_{0}$. At point $B$, i.e. the time $\left(t_{0}+\Delta t_{v}\right)^{-}$, there are,

- The displacement field:

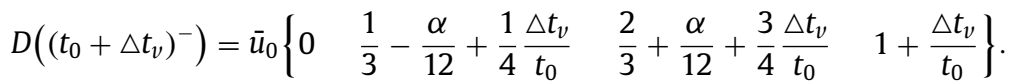

- The VF field stored at time $t_{0}$ :

$$
\mathbf{v}\left(t_{0}, \quad 1, \quad 1, \quad 0, \quad\{\mathrm{E} 4\}\right)=\frac{(1-\alpha) E A \bar{u}_{0}}{6 L}\left\{\begin{array}{llll}
1 & 0 & 1 & 0
\end{array}\right\}
$$

- The internal force field:

$$
\mathbf{F}\left(\left(t_{0}+\Delta t_{v}\right)^{-}\right)=\frac{E A \bar{u}_{0}}{L}\left(\frac{2}{3}-\frac{\alpha}{6}+\frac{1}{2} \frac{\triangle t_{v}}{t_{0}}\right)\left\{\begin{array}{llll}
1 & 0 & 1 & 0
\end{array}\right\} .
$$

- E4's internal force to be released at N2 and N3: $\bar{F}_{\mathrm{E} 4}=\frac{E A \bar{u}_{0}}{L}\left(\frac{1}{3}+\frac{\alpha}{6}+\frac{1}{2} \frac{\Delta t_{v}}{t_{0}}\right)$. 


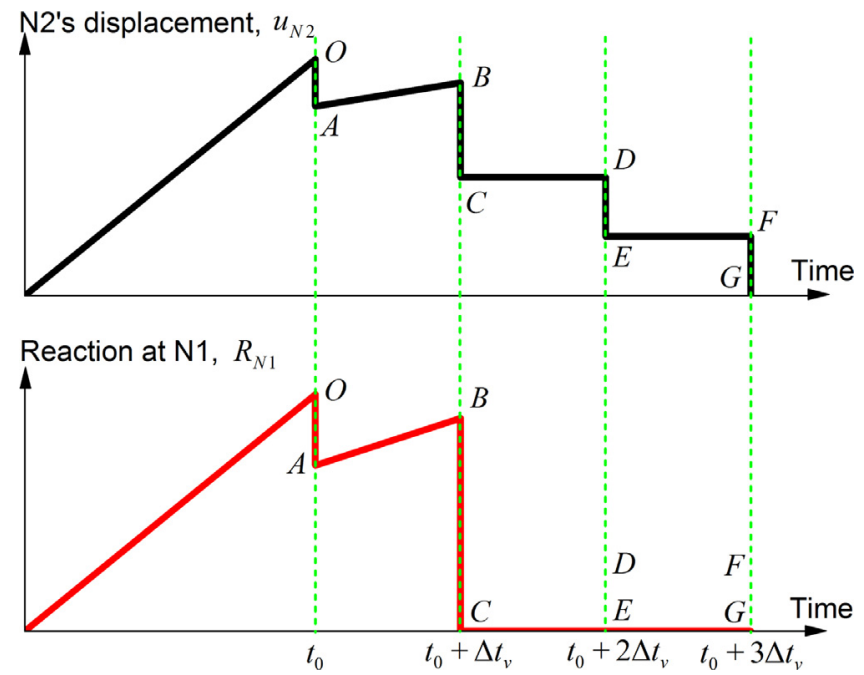

Fig. 12. In case 2 , the curves of the reaction force at $\mathrm{N} 1, R_{\mathrm{N} 1}$, and the displacement of $\mathrm{N} 2, u_{\mathrm{N} 2}$, versus time.

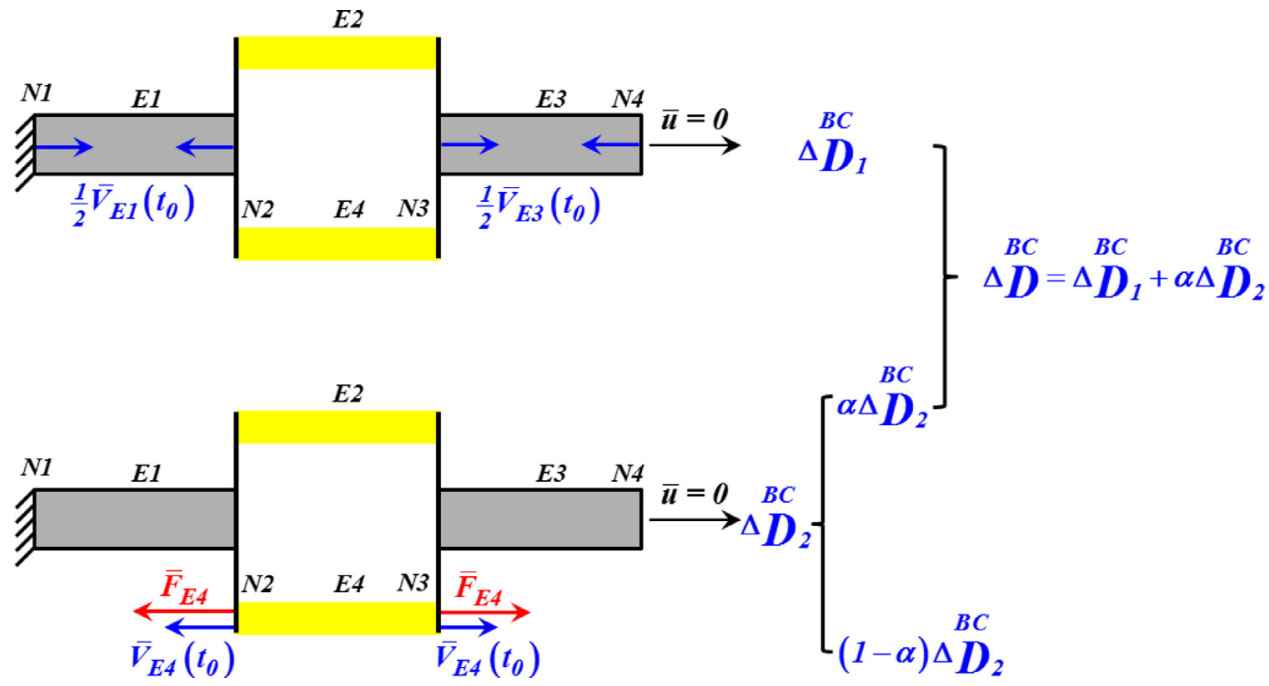

Fig. 13. In case 2, boundary and loading conditions for calculating the incremental deformations, which bridge $B$ and $C$ in Fig. 12 .

- VF in $\mathrm{E} 4$ to be released due to its own rupture: $\bar{V}_{\mathrm{E} 4}\left(t_{0}\right)=\frac{(1-\alpha) E A \bar{u}_{0}}{6 L}$, which is the value in the $\mathrm{VF}$ field $\overline{\mathbf{V}}\left(t_{0}\right)=$ $\mathbf{v}\left(t_{0}, \quad 1, \quad 0, \quad 0, \quad \emptyset\right)$, as shown in Eqs. (3.37) and (3.38).

- E1's VF to be released from $\left(t_{0}+\Delta t_{v}\right)^{-}$to $\left(t_{0}+\Delta t_{v}\right)^{+}$, according to the assumed stepwise viscous relaxation: $\frac{1}{2} \bar{V}_{\mathrm{E} 1}\left(t_{0}\right)=$ $\frac{(1-\alpha) E A \bar{u}_{0}}{12 L}$.

- E3's VF to be released from $\left(t_{0}+\Delta t_{v}\right)^{-}$to $\left(t_{0}+\Delta t_{v}\right)^{+}$, according to the assumed stepwise viscous relaxation: $\frac{1}{2} \bar{V}_{\mathrm{E} 3}\left(t_{0}\right)=$ $\frac{(1-\alpha) E A \bar{u}_{0}}{12 L}$.

- Reaction force at N1: $R\left(\left(t_{0}+\Delta t_{v}\right)^{-}\right)=F_{\mathrm{E} 1}-\bar{V}_{\mathrm{E} 1}\left(t_{0}\right)=\frac{E A \bar{u}_{0}}{L}\left(\frac{1}{6}+\frac{\alpha}{3}+\frac{1}{2} \frac{\Delta t_{v}}{t_{0}}\right)$.

$B \rightarrow C$ process:

There are two kinds of incremental displacement fields to be calculated, as shown in Fig. 13.

The first incremental displacement field is due to the assumed stepwise viscous relaxation, $\frac{1}{2} \bar{V}_{\mathrm{E} 1}\left(t_{0}\right)$ and $\frac{1}{2} \bar{V}_{\mathrm{E} 3}\left(t_{0}\right)$ in intact elements E1 and E3, respectively. It is calculated under the following condition: in the specimen with E2 and E4 eliminated, the controlled displacement at node $\mathrm{N} 4$ is 0 , the forces $\frac{1}{2} \bar{V}_{\mathrm{E} 1}\left(t_{0}\right)(\rightarrow), \frac{1}{2} \bar{V}_{\mathrm{E} 1}\left(t_{0}\right)(\leftarrow), \frac{1}{2} \bar{V}_{\mathrm{E} 3}\left(t_{0}\right)(\rightarrow)$ and $\frac{1}{2} \bar{V}_{\mathrm{E} 3}\left(t_{0}\right)(\leftarrow)$ are applied at nodes N1, N2, N3 and N4, respectively. The incremental field is,

$$
\triangle D_{1}^{B C}=\frac{1-\alpha}{24} \bar{u}_{0}\left\{\begin{array}{llll}
0 & -1 & 1 & 0
\end{array}\right\} .
$$


The second incremental displacement field is due to the release of internal force and VF in E4. It is calculated under the following condition: in the specimen with E2 and E4 eliminated, the controlled displacement at node N4 is 0, the forces $\left(\bar{F}_{\mathrm{E} 4}+\bar{V}_{\mathrm{E} 4}\left(t_{0}\right)\right)(\leftarrow)$ and $\left(\bar{F}_{\mathrm{E} 4}+\bar{V}_{\mathrm{E} 4}\left(t_{0}\right)\right)(\rightarrow)$ are applied at $\mathrm{N} 2$ and $\mathrm{N} 3$, respectively. The corresponding incremental field is,

$$
\triangle D_{2}=\frac{1}{4}\left(1+\frac{\triangle t_{v}}{t_{0}}\right) \bar{u}_{0}\left\{\begin{array}{llll}
0 & -1 & 1 & 0
\end{array}\right\} .
$$

The real incremental displacement field is,

$$
\triangle \stackrel{B C}{D}=\triangle D_{1}^{B C}+\alpha \triangle D_{2}^{B C}=\left(\frac{1}{24}+\frac{5}{24} \alpha+\frac{\alpha}{4} \frac{\Delta t_{v}}{t_{0}}\right) \bar{u}_{0}\left\{\begin{array}{llll}
0 & -1 & 1 & 0
\end{array}\right\} .
$$

Notably, in Fig. 12, the $A \rightarrow C$ process is generally nonlinear, and it is approximately taken as two linear elastic processes, i.e. $A \rightarrow B$ and $B \rightarrow C$ in order to keep the non-iterative feature of the proposed algorithm.

At point $C$, i.e. the time $\left(t_{0}+\Delta t_{v}\right)^{+}$, there are,

- The displacement field:

$$
D\left(\left(t_{0}+\Delta t_{v}\right)^{+}\right)=\bar{u}_{0}\left\{0 \quad \frac{7}{24}-\frac{7 \alpha}{24}+\frac{1-\alpha}{4} \frac{\Delta t_{v}}{t_{0}} \quad \frac{17}{24}+\frac{7 \alpha}{24}+\left(\frac{3}{4}+\frac{\alpha}{4}\right) \frac{\Delta t_{v}}{t_{0}} \quad 1+\frac{\Delta t_{v}}{t_{0}}\right\} .
$$

- The VF field stored at $t_{0}$ :

$$
\mathbf{v}\left(t_{0}, \quad 2, \quad 0, \quad 0, \quad\{\mathrm{E} 4\}\right)=\frac{E A \bar{u}_{0}(1-\alpha)}{12 L}\left\{\begin{array}{llll}
1 & 0 & 1 & 0
\end{array}\right\}
$$

- The VF field stored at $t_{0}+\Delta t_{v}$ :

$$
\mathbf{v}\left(t_{0}+\Delta t_{v}, \quad 1, \quad 0, \quad 0, \quad \emptyset\right)=\frac{E A \bar{u}_{0}(1-\alpha)}{2 L}\left(1+\frac{\Delta t_{v}}{t_{0}}\right)\left\{\begin{array}{llll}
1 & 0 & 1 & 0
\end{array}\right\},
$$

which is obtained in $-(1-\alpha) \triangle D_{2}$. For the convenience of the following statement, note

$$
\overline{\mathbf{v}}\left(t_{0}+\Delta t_{v}\right)=\mathbf{v}\left(t_{0}+\Delta t_{v}, \quad 1, \quad 0, \quad 0, \quad \emptyset\right) .
$$

- The internal force field:

$$
\mathbf{F}\left(\left(t_{0}+\Delta t_{v}\right)^{+}\right)=\frac{E A \bar{u}_{0}(1-\alpha)}{L}\left(\frac{7}{12}+\frac{1}{2} \frac{\Delta t_{v}}{t_{0}}\right)\left\{\begin{array}{llll}
1 & 0 & 1 & 0
\end{array}\right\} .
$$

- There is no more internal force to be released.

- There is no more VF to be released at this time.

- Reaction force at N1: $R\left(\left(t_{0}+\Delta t_{v}\right)^{+}\right)=F_{\mathrm{E} 1}-\frac{1}{2} \bar{V}_{\mathrm{E} 1}\left(t_{0}\right)-\frac{1}{2} \bar{V}_{\mathrm{E} 1}\left(t_{0}+\Delta t_{v}\right)=0$.

$C \rightarrow D$ process:

The corresponding incremental displacement field is calculated under the following condition: in the specimen with E2 and E4 eliminated, the controlled displacement at node N4 is $\frac{\Delta t_{v}}{t_{0}} \bar{u}_{0}$.

At point $D$, i.e. the time $\left(t_{0}+2 \triangle t_{v}\right)^{-}$, there are,

- The displacement field:

$$
D\left(\left(t_{0}+2 \Delta t_{v}\right)^{-}\right)=\bar{u}_{0}\left\{0 \quad \frac{7}{24}-\frac{7 \alpha}{24}+\frac{1-\alpha}{4} \frac{\Delta t_{v}}{t_{0}} \quad \frac{17}{24}+\frac{7 \alpha}{24}+\left(\frac{7}{4}+\frac{\alpha}{4}\right) \frac{\Delta t_{v}}{t_{0}} \quad 1+2 \frac{\Delta t_{v}}{t_{0}}\right\} .
$$

- The VF field stored around $t_{0}$ :

$$
\mathbf{v}\left(t_{0}, \quad 2, \quad 1, \quad 0, \quad\{\mathrm{E} 4\}\right)=\frac{(1-\alpha) E A \bar{u}_{0}}{12 L}\left\{\begin{array}{llll}
1 & 0 & 1 & 0
\end{array}\right\}
$$

- The VF field stored around $t_{0}+\Delta t_{v}$ :

$$
\mathbf{v}\left(t_{0}+\Delta t_{v}, \quad 1, \quad 1, \quad 0, \quad \emptyset\right)=\frac{E A \bar{u}_{0}(1-\alpha)}{2 L}\left(1+\frac{\Delta t_{v}}{t_{0}}\right)\left\{\begin{array}{llll}
1 & 0 & 1 & 0
\end{array}\right\} .
$$

- The internal force field:

$$
\mathbf{F}\left(\left(t_{0}+2 \triangle t_{v}\right)^{-}\right)=\frac{E A \bar{u}_{0}(1-\alpha)}{L}\left(\frac{7}{12}+\frac{1}{2} \frac{\Delta t_{v}}{t_{0}}\right)\left\{\begin{array}{llll}
1 & 0 & 1 & 0
\end{array}\right\} .
$$

- E1's VF to be released from $\left(t_{0}+2 \Delta t_{v}\right)^{-}$to $\left(t_{0}+2 \Delta t_{v}\right)^{+}$, according to the assumed stepwise viscous relaxation: $\frac{1}{2} \bar{V}_{\mathrm{E} 1}\left(t_{0}\right)+\frac{1}{2} \bar{V}_{\mathrm{E} 1}\left(t_{0}+\Delta t_{v}\right)$.

- E3's VF to be released from $\left(t_{0}+2 \Delta t_{v}\right)^{-}$to $\left(t_{0}+2 \Delta t_{v}\right)^{+}$, according to the assumed stepwise viscous relaxation: $\frac{1}{2} \bar{V}_{\mathrm{E} 3}\left(t_{0}\right)+\frac{1}{2} \bar{V}_{\mathrm{E} 3}\left(t_{0}+\Delta t_{v}\right)$.

- Reaction force at $\mathrm{N} 1: R\left(\left(t_{0}+2 \Delta t_{v}\right)^{-}\right)=F_{\mathrm{E} 1}-\frac{1}{2} \bar{V}_{\mathrm{E} 1}\left(t_{0}\right)-\bar{V}_{\mathrm{E} 1}\left(t_{0}+\Delta t_{v}\right)=0$. 


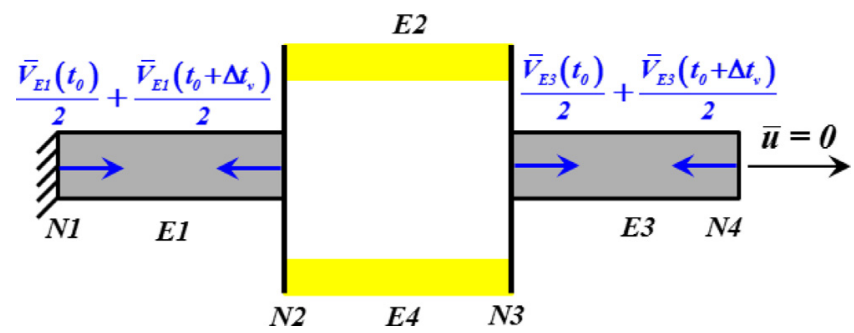

Fig. 14. In case 2, boundary and loading conditions for calculating the incremental deformation, which bridges $D$ and $E$ as shown in Fig. 12 .

$D \rightarrow$ E process:

The incremental displacement field is due to the assumed stepwise viscous relaxation, $\frac{1}{2} \bar{V}_{\mathrm{E} 1}\left(t_{0}\right), \frac{1}{2} \bar{V}_{\mathrm{E} 3}\left(t_{0}\right), \frac{1}{2} \bar{V}_{\mathrm{E} 1}\left(t_{0}+\Delta t_{v}\right)$ and $\frac{1}{2} \bar{V}_{\mathrm{E} 3}\left(t_{0}+\Delta t_{v}\right)$. Refer to Fig. 14, it is calculated under the following condition: in the specimen with E2 and E4 eliminated, the controlled displacement at node $\mathrm{N} 4$ is 0 , the forces $\frac{1}{2}\left[\bar{V}_{\mathrm{E} 1}\left(t_{0}\right)+\bar{V}_{\mathrm{E} 1}\left(t_{0}+\Delta t_{v}\right)\right](\rightarrow), \frac{1}{2}\left[\bar{V}_{\mathrm{E} 1}\left(t_{0}\right)+\bar{V}_{\mathrm{E} 1}\left(t_{0}+\Delta t_{v}\right)\right](\leftarrow)$, $\frac{1}{2}\left[\bar{V}_{\mathrm{E} 3}\left(t_{0}\right)+\bar{V}_{\mathrm{E} 3}\left(t_{0}+\Delta t_{v}\right)\right](\rightarrow)$ and $\frac{1}{2}\left[\bar{V}_{\mathrm{E} 3}\left(t_{0}\right)+\bar{V}_{\mathrm{E} 3}\left(t_{0}+\Delta t_{v}\right)\right](\leftarrow)$ are applied at N1, N2, N3 and N4, respectively. The incremental field is,

$$
\triangle \stackrel{D E}{D}=\left(\frac{1}{3}+\frac{1}{4} \frac{\Delta t_{v}}{t_{0}}\right) \frac{1-\alpha}{2} \bar{u}_{0}\left\{\begin{array}{llll}
0 & -1 & 1 & 0
\end{array}\right\} .
$$

At point $E$, i.e. the time $\left(t_{0}+2 \Delta t_{v}\right)^{+}$, there are,

- The displacement field:

$$
D\left(\left(t_{0}+2 \triangle t_{v}\right)^{+}\right)=\bar{u}_{0}\left\{0 \quad(1-\alpha)\left(\frac{1}{8}+\frac{1}{8} \frac{\triangle t_{v}}{t_{0}}\right) \quad \frac{7}{8}+\frac{\alpha}{8}+\left(\frac{15}{8}+\frac{\alpha}{8}\right) \frac{\Delta t_{v}}{t_{0}} \quad 1+2 \frac{\Delta t_{v}}{t_{0}}\right\} .
$$

- The VF field stored around $t_{0}$ :

$$
\mathbf{v}\left(t_{0}, \quad 2, \quad 1, \quad 1, \quad\{E 4\}\right)=\left\{\begin{array}{llll}
0 & 0 & 0 & 0
\end{array}\right\}
$$

which has been fully released. It vanishes from $\left(t_{0}+2 \Delta t_{v}\right)^{+}$on.

- The VF field stored around $t_{0}+\Delta t_{v}$ :

$$
\mathbf{v}\left(t_{0}+\Delta t_{v}, \quad 2, \quad 0, \quad 0, \quad \emptyset\right)=\frac{E A \bar{u}_{0}(1-\alpha)}{4 L}\left(1+\frac{\Delta t_{v}}{t_{0}}\right)\left\{\begin{array}{llll}
1 & 0 & 1 & 0
\end{array}\right\} .
$$

- The internal force field:

$$
\mathbf{F}\left(\left(t_{0}+2 \triangle t_{v}\right)^{+}\right)=\frac{E A \bar{u}_{0}(1-\alpha)}{4 L}\left(1+\frac{\triangle t_{v}}{t_{0}}\right)\left\{\begin{array}{llll}
1 & 0 & 1 & 0
\end{array}\right\} .
$$

- There is no more internal force to be released.

- There is no more VF to be release at this time.

- Reaction force at N1: $R\left(\left(t_{0}+2 \Delta t_{v}\right)^{+}\right)=F_{\mathrm{E} 1}-\frac{1}{2} \bar{V}_{\mathrm{E} 1}\left(t_{0}+\Delta t_{v}\right)=0$.

$E \rightarrow F$ process:

The incremental displacement field is calculated under the following condition: in the specimen with E2 and E4 eliminated, the controlled displacement on node N4 is $\frac{\Delta t_{v}}{t_{0}} \bar{u}_{0}$.

At point $F$, i.e. the time $\left(t_{0}+3 \Delta t_{v}\right)^{-}$, there are,

- The displacement field:

$$
D\left(\left(t_{0}+3 \triangle t_{v}\right)^{-}\right)=\bar{u}_{0}\left\{0 \quad(1-\alpha)\left(\frac{1}{8}+\frac{1}{8} \frac{\Delta t_{v}}{t_{0}}\right) \quad \frac{7}{8}+\frac{\alpha}{8}+\left(\frac{23}{8}+\frac{\alpha}{8}\right) \frac{\Delta t_{v}}{t_{0}} \quad 1+3 \frac{\Delta t_{v}}{t_{0}}\right\} .
$$

- The viscous force field stored around $t_{0}+\Delta t_{v}$ :

$$
\mathbf{v}\left(t_{0}+\Delta t_{v}, \quad 2, \quad 1, \quad 0, \quad \emptyset\right)=\frac{E A \bar{u}_{0}(1-\alpha)}{4 L}\left(1+\frac{\Delta t_{v}}{t_{0}}\right)\left\{\begin{array}{llll}
1 & 0 & 1 & 0
\end{array}\right\} .
$$

- The internal force field:

$$
\mathbf{F}\left(\left(t_{0}+3 \triangle t_{v}\right)^{-}\right)=\frac{E A \bar{u}_{0}(1-\alpha)}{4 L}\left(1+\frac{\Delta t_{v}}{t_{0}}\right)\left\{\begin{array}{llll}
1 & 0 & 1 & 0
\end{array}\right\} .
$$

- E1's VF to be release from $\left(t_{0}+3 \Delta t_{v}\right)^{-}$to $\left(t_{0}+3 \Delta t_{v}\right)^{+}$, according to the assumed stepwise viscous relaxation: $\frac{1}{2} \bar{V}_{\mathrm{E} 1}\left(t_{0}+\Delta t_{v}\right)$ 
- E3's VF to be release from $\left(t_{0}+3 \Delta t_{v}\right)^{-}$to $\left(t_{0}+3 \Delta t_{v}\right)^{+}$, according to the assumed stepwise viscous relaxation: $\frac{1}{2} \bar{V}_{\mathrm{E} 3}\left(t_{0}+\Delta t_{v}\right)$.

- Reaction force at $\mathrm{N} 1: R\left(\left(t_{0}+3 \Delta t_{v}\right)^{-}\right)=F_{\mathrm{E} 1}-\frac{1}{2} \bar{V}_{\mathrm{E} 1}\left(t_{0}+\Delta t_{v}\right)=0$.

$F \rightarrow G$ process:

The incremental displacement field is due to the assumed stepwise viscous relaxation, $\frac{1}{2} \bar{V}_{\mathrm{E} 1}\left(t_{0}+\Delta t_{v}\right)$ and $\frac{1}{2} \bar{V}_{\mathrm{E} 3}\left(t_{0}+\Delta t_{v}\right)$. It is calculated under the following condition: in the specimen with E2 and E4 eliminated, the controlled displacement at node $\mathrm{N} 4$ is 0 , the forces $\frac{1}{2} \bar{V}_{\mathrm{E} 1}\left(t_{0}+\Delta t_{v}\right)(\rightarrow), \frac{1}{2} \bar{V}_{\mathrm{E} 1}\left(t_{0}+\Delta t_{v}\right)(\leftarrow), \frac{1}{2} \bar{V}_{\mathrm{E} 3}\left(t_{0}+\Delta t_{v}\right)(\rightarrow)$ and $\frac{1}{2} \bar{V}_{\mathrm{E} 3}\left(t_{0}+\Delta t_{v}\right)(\leftarrow)$ are applied at $\mathrm{N} 1, \mathrm{~N} 2, \mathrm{~N} 3$ and N4, respectively.

At point $G$, i.e. the time $\left(t_{0}+3 \Delta t_{v}\right)^{+}$, there are,

- The displacement field:

$$
D\left(\left(t_{0}+3 \triangle t_{v}\right)^{+}\right)=\left(1+3 \frac{\Delta t_{v}}{t_{0}}\right) \bar{u}_{0}\left\{\begin{array}{llll}
0 & 0 & 1 & 1
\end{array}\right\} .
$$

- The VF field stored around $t_{0}+\Delta t_{v}$ :

$$
\mathbf{v}\left(t_{0}+\Delta t_{v}, \quad 2, \quad 1, \quad 1, \quad \emptyset\right)=\left\{\begin{array}{llll}
0 & 0 & 0 & 0
\end{array}\right\} .
$$

- The internal force field:

$$
\mathbf{F}\left(\left(t_{0}+2 \triangle t_{v}\right)^{+}\right)=\left\{\begin{array}{llll}
0 & 0 & 0 & 0
\end{array}\right\} .
$$

- There is no more internal force to be released.

- There is no more VF to be released since then.

- Reaction force at $\mathrm{N} 1: R\left(\left(t_{0}+2 \triangle t_{v}\right)^{+}\right)=0$.

\section{Event-driven algorithm associated with elastoviscous stress redistribution}

The progressive failure under external loading is simulated by successive occurrences of "events", which may be breakage of intact elements, contact/separation of previously cracked elements, or step-wise releasing of critical viscous force fields. Thus, during the performance of a numerical simulation it is necessary to detect new event(s) correctly. The event-driven algorithm (refer to [16,37,38]), which is also called the event-by-event method, is adopted here. In each step, the value of increment in load or forces to be released depends on the appearance of new critical element(s).

\subsection{Categories of critical events}

After applying a trial increment, the normal stress $\sigma$ and the shear stress $\tau$ in each beam are compared with the strength surface, which has been defined by Eq. (2.2) and shown in Fig. 2.

Additionally, as shown in Fig. 3, the separation/contact of cracked elements is detected according to

$$
\begin{aligned}
& \frac{\sqrt{(\Delta u)^{2}+(L+\Delta v)^{2}}}{L}>1 \quad(\text { contact } \rightarrow \text { separation }), \\
& \left.\frac{L}{\sqrt{(\Delta u)^{2}+(L+\Delta v)^{2}}}>1 \quad \text { (separation } \rightarrow \text { contact }\right),
\end{aligned}
$$

where, $\Delta u=u_{j}-u_{i}$ and $\Delta v=v_{j}-v_{i}$ are the relative displacements between ends $i$ and $j$ in an element. As for step-wise release of VF fields, the present state of the VF field stored at time $t_{0}$ is expressed as $\mathbf{v}\left(t_{0}, \quad n_{v}, \quad \phi_{t}, \quad \phi_{v}, \quad \mathbb{R}\right)$. We need to check whether the present time, $t$, has reached the value of $t_{0}+\sum_{i=1}^{n_{v}} \Delta t_{i}$, and the rule of judging the $n_{v}$ th VF step can be expressed as,

$$
\frac{t}{t_{0}+\sum_{i=1}^{n_{v}} \Delta t_{i}}=1 .
$$

Only one event is permitted per computational cycle. If several elements and/or VF fields have violated strength rules, the most critical one undergoes a new event.

Treatments of the above different events are listed in Table 1. Notably, for both breakage of intact element and step-wise release of VF field will lead to stress redistribution (or equivalently relaxation), for which the relevant strategy involved is illustrated in Section 3. 
Table 1

Summary of event types and the corresponding computational treatments in the present event-driven algorithm.

\begin{tabular}{llcl}
\hline Treatment\Event & Breakage of intact element & Seperation/contact of cracked element & Stepwise release of VF field \\
\hline Stiffness adjustment & $\checkmark$ & $\checkmark$ & - \\
Stress redistribution & $\checkmark$ & - & $\checkmark$ \\
VF field storage & $\checkmark$ & - & - \\
Induced event check & $\checkmark$ & - & $\checkmark$ \\
\hline
\end{tabular}

\section{Start from an initial state}
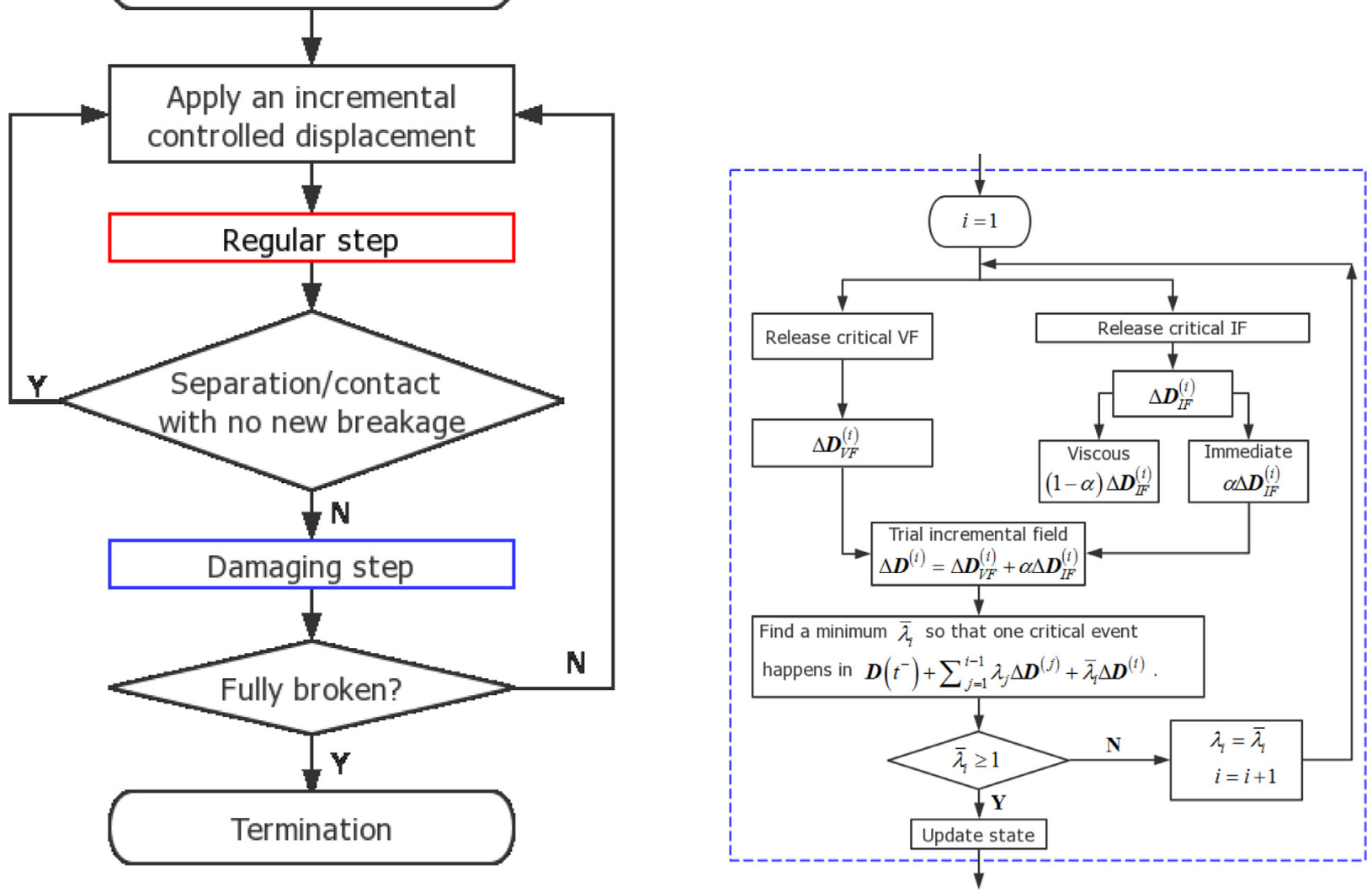

a

c

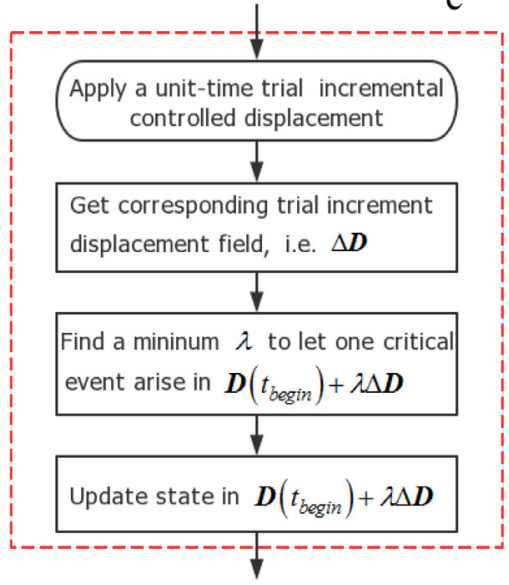

b

Fig. 15. Flow charts of (a) the whole event-driven algorithm, (b) a regular step and (c) a damaging step. 


\subsection{Simulation procedure}

The simulation is implemented by sequentially detecting and treating critical events. The flow chart of such an eventdriven algorithm is shown in Fig. 15(a). According to whether the external controlled displacement varies, two kinds of incremental steps are proposed. One is the so-called "regular steps" where there is no more force to be released at the very beginning of the step and a new critical event can only appear by letting time elapse and/or external controlled loading vary, and the other kind is the so-called "damaging step" where some viscous and/or internal forces need to be released immediately, preventing time and external controlled displacement from evolving before their full release. Specifically, when the critical event detected during a regular step is contact/separation of cracked element with no new breakage, there will be no forces to be released at the step end, and therefore the next step is still a regular one.

For a regular step (Fig. 15(b)), a trial increment in controlled displacement is applied, which produces the displacement field $\Delta D$. Then, a minimum $\lambda$ is determined so that just one critical event arises in the field $D_{0}+\lambda \Delta D$. Notably, in this study, events occurring under the same $\lambda$ are merged as one single "critical event". Then, according to the type of "critical event", the specimen state should be updated correspondingly, as shown in Table 1. The state-updating operation will be presented in more details at the end of this section.

A damaging step can be much more complex than a regular one, due to possible occurrences of sequentially induced failures, as shown in Fig. 15(c). With reference to Fig. 15(a), there must be some viscous and/or internal forces to be released. Based on the assumption already mentioned in Section 3, the release of both internal forces and stepwise VF fields (refer to Fig. 11) is infinitely faster than the external loading rate. As a result, the damaging step is deemed taking no time to happen. In Fig. 15(c), the release of VF field leads to the incremental displacement field $\Delta D_{V F}^{(i)}$, which is assumed to be completely an immediate elastic process. While the release of internal forces (IF) gives rise to $\Delta D_{I F}^{(i)}$, which is composed of an immediate part, i.e. $\alpha \Delta D_{I F}^{(i)}$, and a viscous part, i.e. $(1-\alpha) \Delta D_{I F}^{(i)}$, that actually does not take place presently and is substituted with a corresponding VF field (e.g., refer to Fig. 13 for illustration). Here, the superscript " $i$ ", which is initially set as 1 at the beginning of the damaging step, stands for the iteration numbering for sequentially checking induced critical events. Therefore, the trial incremental field at the present iteration can be written as

$$
\Delta D^{(i)}=\Delta D_{V F}^{(i)}+\alpha \Delta D_{I F}^{(i)},
$$

where, on the right-hand side, the first term is the contribution of VF release, while the second term is due to the IF release. Notably, in Section 3, a simplified notation has been adopted, i.e. $\lambda_{1}$, which is the same as $\lambda^{(1)}$ here. So are $\Delta D^{(i)}$ and $\Delta D_{i}$. Then an operation of detecting induced events (refer to Section 4.1) is conducted, i.e. finding a minimum $\bar{\lambda}^{(i)}$ so that one critical event happens in the total displacement field,

$$
\bar{D}^{(i)}(t)=D\left(t^{-}\right)+\sum_{j=1}^{i-1} \lambda^{j} \Delta D^{(j)}+\bar{\lambda}^{(i)} \Delta D^{(i)},
$$

where, the "-" in $\bar{D}^{(i)}(t)$ and $\bar{\lambda}^{(i)}$ indicates the corresponding variables are "trial" in nature, and they can become real only when $\bar{\lambda}^{(i)} \leqslant 1$. The above iteration is stopped once $\bar{\lambda}^{(i)}>1$.

It is important to list what need be done in the "update state" operation, which appears in both regular and damaging steps, as shown in Fig. 15(b) and (c):

- The present displacement field, may be generally written as $D\left(t_{\text {begin }}\right)+\sum_{i} \lambda^{(i)} \Delta D^{(i)}$. For a regular step, $i=1$, therefore it becomes $D\left(t_{\text {begin }}\right)+\lambda \Delta D$, where $\Delta D$ is produced by applying a trial increment in the controlled displacement. For a damaging step, due to the possibility of sequential induced failures, it may take more than one iteration to reach the final equilibrium. Each iteration makes a contribution of $\lambda^{i} \Delta D^{(i)}$. Particularly, now $t_{\text {begin }}$ becomes $t^{-}$.

- Updating of previously stored VF fields. If some step among a VF field previously stored is to be released now, the remaining part is $\prod_{i}\left(1-\lambda^{(i)}\right)$ times of the whole magnitude of this critical step. It has been fully released after the latest $\lambda^{(i)}=1$, leading to $\prod_{i}\left(1-\lambda^{(i)}\right)=0$.

- Treatment of VF fields previously stored in elements induced to break presently. All VFs remaining in it should be summed up and taken as "internal forces" to be released immediately once and for all.

- Calculation of internal forces in intact elements. It needs to be done in the displacement field $D\left(t_{\text {begin }}\right)+\sum_{i} \lambda^{(i)} \Delta D^{(i)}$ by using the present elemental stiffness.

- Evolution of internal forces to be released immediately. There are two kinds of forces included, i.e. internal forces so far stored in the newly broken elements, and viscous forces in them, as just mentioned above.

- Updating of VF field presently being stored. New VF field can arise only in damaging steps. During a damaging step, the newly arising VF is calculated in the displacement field $\sum_{i}(1-\alpha) \lambda^{(i)} \Delta D_{I F}^{(i)}$.

\section{Numerical examples and analyses}

The proposed damage event-driven model based on the concept of VF field is used to simulate a series of failure phenomena in order to show its effectiveness of capturing main characteristics in quasi-brittle failures. 


\section{Table 2}

Parameter setting related to elasticity, strength and failure-induced viscosity. Notes: for convenience, $\Delta t_{v 1}$ is taken as the basic material parameter. The controlled loading velocity $\bar{v}$ is measured by relating it to $\Delta t_{v 1}$ via $\bar{v}=\tilde{d} / \Delta t_{v 1}$. Here the coefficient $\tilde{d}$ means the displacement under the velocity $\bar{v}$ in time $\Delta t_{v 1}$. In Case 1 , we let $\bar{v} \cdot \Delta t_{v 1}=\tilde{d}=5.0 \times 10^{-7} \mathrm{~m}$, a uniform controlled displacement along the horizontal direction is applied to produce a macro-level uniaxial tension. From a pure computational viewpoint, the value of $\tilde{d}$ influences the result, while $\bar{v}$ and $\Delta t_{v 1}$ are easily determined once either is known. For example, when we set $\Delta t_{v 1}=0.01 \mathrm{~s}, \bar{v}$ becomes $5.0 \times 10^{-5} \mathrm{~m} / \mathrm{s}$. The average strain rate along the tensile direction is $\dot{\bar{\varepsilon}}=\tilde{d} / l \Delta t_{v 1}$, where $l$ is the sidelength of the rectangular specimen along the tested direction. In (a), $D=0.9$ means that the contact stiffness of cracked element is $(1-D)$ times of the intact normal stiffness. In (b), proportions of VF released in VF step no. $0-4$ is $1: 1: 1: 1: 1$, thus the parameter $\alpha$ defined in Section 3 is equal to 0.2 by keeping in mind that step no. 0 corresponds to the immediate elasticity.

(a) Meso elastic and strength properties of the material.

\begin{tabular}{|c|c|c|c|c|c|c|}
\hline Phases \Properties & $E(\mathrm{MPa})$ & $f_{t}(\mathrm{MPa})$ & $f_{c}(\mathrm{MPa})$ & $c(\mathrm{MPa})$ & $\phi\left(^{\circ}\right)$ & $D$ \\
\hline Aggregate & 70,000 & 10.0 & 120.0 & 15.0 & 45 & 0.9 \\
\hline Interface & 25,000 & 2.7 & 15.0 & 1.875 & 45 & 0.9 \\
\hline Matrix & 25,000 & 5 & 60.0 & 7.5 & 45 & 0.9 \\
\hline
\end{tabular}

(b) The settings related to viscosity induced by elemental breakage adopted for modeling.

\begin{tabular}{lll}
$\begin{array}{l}\text { Failure induced } \\
\text { viscosity }\end{array}$ & Viscous force field caused by each & $\Delta t_{v 1}: \Delta t_{v 2}: \Delta t_{v 3}: \Delta t_{v 4}=1: 2: 4: 8$ \\
& elemental breakage takes 5 steps to & \\
& release, with step no. 0 immediately \\
& elastic and $1-4$ viscus. & VF released in step no. $0-4: 1: 1: 1: 1: 1$ \\
\hline
\end{tabular}

The tensile tests are performed on a GB lattice with a rectangular geometry of 9.75 by $9.52 \mathrm{~cm}$. The employed lattice is triangular and has a total of 10,066 GB elements and 3433 nodes. All elements have a common length, i.e. $\sqrt{3} / 10 \mathrm{~cm}$. The load is applied to the lattice by setting a uniform controlled displacement at the right edge and fixing the left edge.

A total of 9 cases are simulated in order to explore failure characteristics from various viewpoints.

The first case, i.e. Case 1, has the following settings:

- The parameters associated with meso-scale elastic, strength and failure-induced viscosity are listed in Table 2.

- The loading (right) and constrained (left) edges have matrix-phase elastic properties, while its strength is 100 times of that of the matrix material. All horizontal translational degrees of freedom are fixed on the left edge, and with a velocity given by $\bar{v} \cdot \Delta t_{v 1}=\tilde{d}=5.0 \times 10^{-7} \mathrm{~m}$ on the right edge, where $\Delta t_{v 1}$ is considered as material-dependent and it is set as $0.01 \mathrm{~s}$ without loss of generality.

- On the upper and bottom edges, no constraint is added.

- A particle overlay including 150 aggregates of different sizes is employed. Aggregates are randomly projected onto the matrix-phase lattice, which results in a random three-phased mesostructure composing of aggregate, matrix and interfaces between them.

Case 2 is the same as Case 1 except that the viscous effect is excluded. Namely, it is simulated by the previous quasistatic lattice model $[13,37,39]$. Now we have $\bar{v} \cdot \Delta t_{v 1} \rightarrow 0$. Since we have fixed $\Delta t_{v 1}=0.01 \mathrm{~s}$, there is $\bar{v} \rightarrow 0$, which indicates a quasi-static loading condition.

Case 3 is the same as Case 1 except that the loading rate $\bar{v}$ is 0.01 times of that in Case 1, i.e. $\bar{v}=5.0 \times 10^{-7} \mathrm{~m} / \mathrm{s}$.

Case 4 is the same as Case 1 except that the loading rate $\bar{v}$ is 0.1 times of that in Case 1 , i.e. $\bar{v}=5.0 \times 10^{-6} \mathrm{~m} / \mathrm{s}$.

Case 5 is the same as Case 1 except that the loading rate $\bar{v}$ is 10 times of that in Case 1 , i.e. $\bar{v}=5.0 \times 10^{-4} \mathrm{~m} / \mathrm{s}$.

Case 6 is the same as Case 1 except that $\bar{v}$ is equal to that in Case 1 before the controlled displacement reaches $7 \mu m$, and it is 0.01 times of that in Case 1 when the controlled displacement is increased from $7 \mu \mathrm{m}$ to $9 \mu \mathrm{m}$. Then, $\bar{v}$ is equal to that in Case 1 again.

Case 7 is the same as Case 1 except that each VF field is released in 5 VF steps with the ratio of 6: 1: 1: 1: 1. Considering that the 0th stepwise release is the force field immediately released upon the occurrence of elemental breakage, the bigger its proportion is, the more profound the immediate elasticity is among the stress-redistribution deformation.

Case 8 is the same as Case 3 except that $\Delta t_{v 2}, \Delta t_{v 3}$ and $\Delta t_{v 4}$ are set as $10^{8} \Delta t_{v 1}$, indicating that the last $60 \%$ of the viscous force field will not be released within the interested time range which is far smaller than $10^{8} \Delta t_{v 1}$. As a result, it leads to a permanent deformation.

Case 9 is the same as Case 1 except that the controlled displacement is applied in a cyclic manner. The absolute value of loading rate is kept constant, but the loading direction is reversed cyclicly.

\subsection{Analyses of a typical result compared with the previous lattice model}

To show the characteristics of progressive failures associated with the time-dependent stress redistribution, the results of the present model, i.e. Case 1, are compared with those produced via the previous model without any temporal effect, i.e. Case 2, which are shown in Fig. 16. 

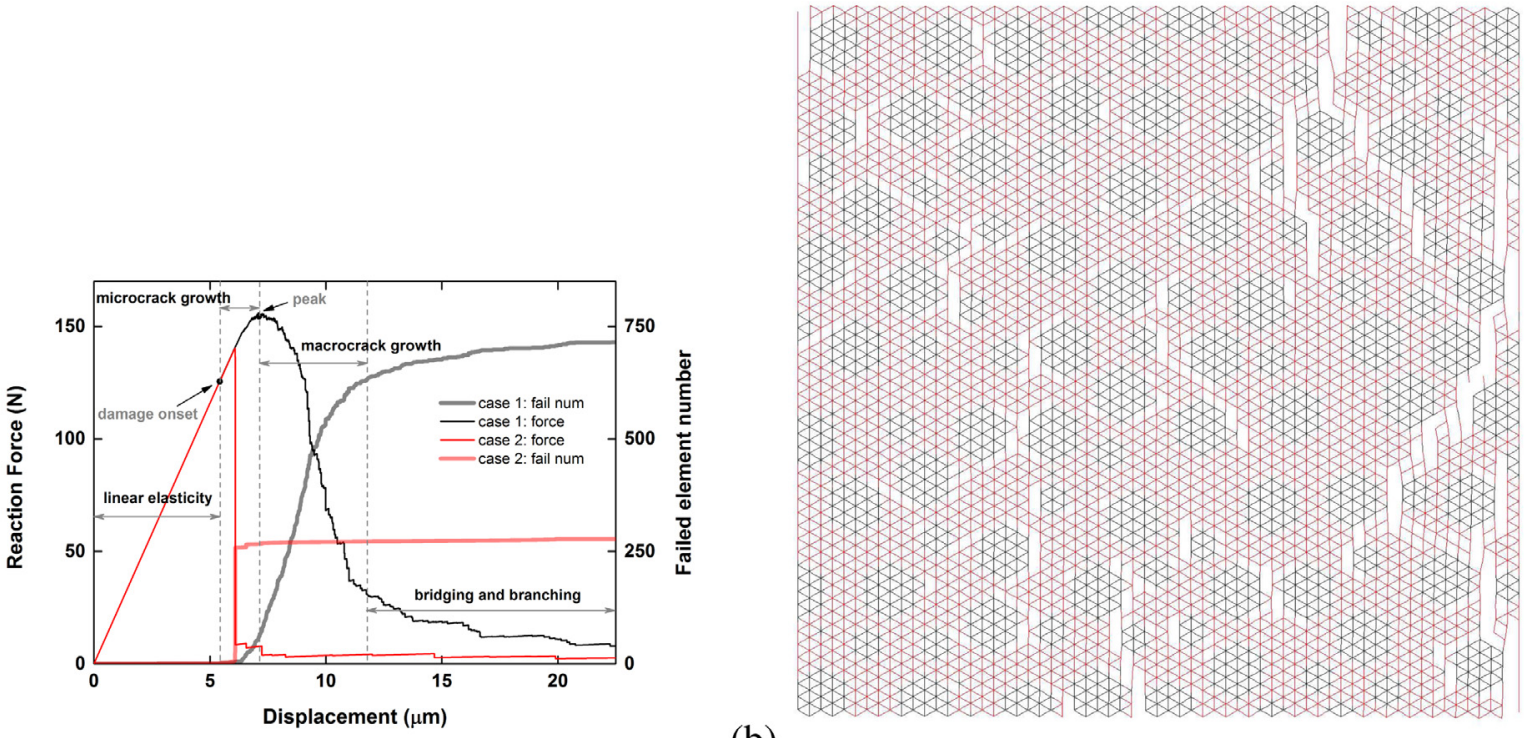

(a)

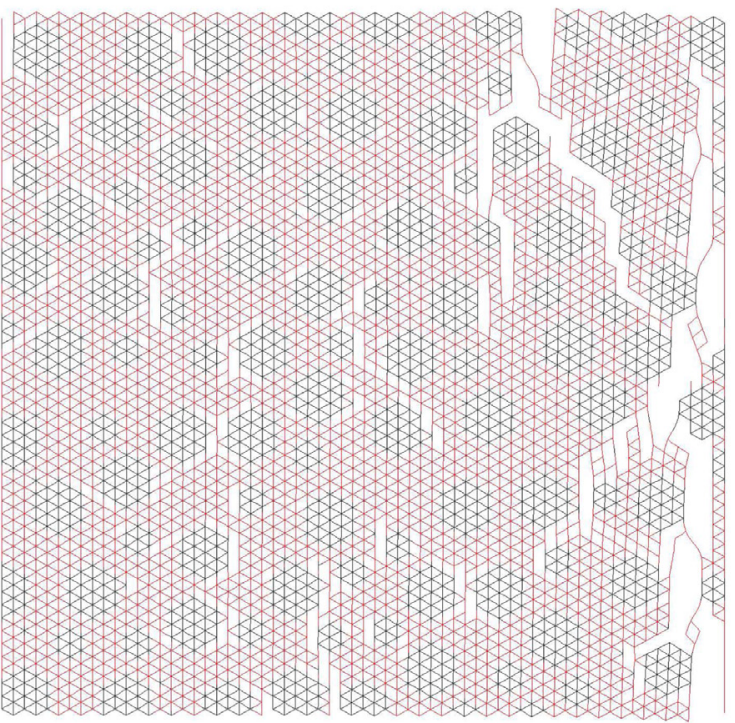

(c)

(d) (b)

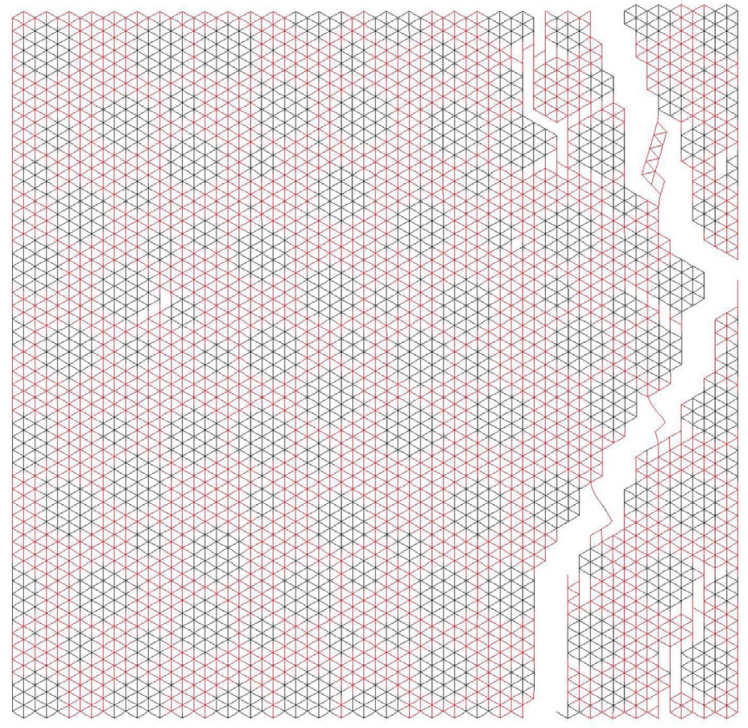

Fig. 16. Results of a typical example, i.e. Case 1, which are compared with Case 2. (a) Diagrams of reaction force and accumulated broken element numbers versus the controlled displacement. Crack patterns corresponding to the controlled displacement (b) $8.20 \mu \mathrm{m}$, (c) $20.0 \mu \mathrm{m}$ in Case 1 , and (d) $6.10 \mu \mathrm{m}$ in Case 2.

In this study we simply consider the viscous effect immediately after the occurrence of local failures. Thus naturally the first elemental breakage happens in the same element and at the same loading level, i.e. when the controlled displacement $\bar{u}=5.43 \mu \mathrm{m}$.

There is a nonlinear stage between the first elemental failure and the peak force. The peak force for Case 1 is $156 \mathrm{~N}$, which is much larger than that for Case 2, $140 \mathrm{~N}$. Meanwhile its nonlinear stage is more lasting than that in Case 2: from Fig. 16(a), the controlled displacement span during the nonlinear stage in Case 1 is equal to $1.57 \mu \mathrm{m}$, which is obviously larger than that in Case 2, i.e. $0.64 \mu \mathrm{m}$.

The viscosity associated with local ruptures also influences the post-peak softening behaviors. In Case 2 where there is no viscosity, the post-peak ductility is very weak. Actually, this has been a fundamental issue in lattice modeling for a few decades $[11,20,21,24]$. If the time-dependence in stress redistribution is not considered, the post-peak ductility remains unrealistically low whether the force-release or load-unload algorithm is adopted [11,13,20,21]. Nevertheless, when viscosity is considered, i.e. in Case 1, the load-displacement curve is much more realistic and is closer to experimental observations presented by Prado et al. [40]. This is explained as follows. The local failure influences the remaining specimen in two 
ways: (i) decreasing the total stiffness due to the vanishing stiffness of the broken element, and (ii) induced relaxation of the remaining specimen for the forces previously stored in the broken element need to be released. The longer the stress redistribution process takes, the less possible other intact elements will be induced to break. In Case 2, the stress redistribution takes no time to complete immediately after the onset of local breakage, which leads to the over-brittle issue $[11,21]$.

Furthermore, viscosity plays a considerable role in the progressive crack patterns, which is analyzed during the pre- and post-peak stage in the following:

- The pre-peak behaviors are similar in both cases. Elemental breakages keep accumulating with increasing external loading, and no severe macro cracks appear. Refer to Fig. 15(a), during the pre-peak period, the total number of broken elements is 60 in Case 1, but only 7 in Case 2.

- The post-peak crack patterns are strongly influenced by viscosity. In Case 2 with no viscosity, immediately after the peak, the deformation in the entire specimen is highly localized at the macro-level. In other words, a macrocrack appears, which is corresponding to both the steep drop in reaction force and the sudden increase in broken element number, as shown in Fig. 16(a). While in Case 1 where viscosity is accounted for, immediately after the peak, more scattering elemental breakages arise throughout the specimen without severe localization. In other words, macro-cracks dot not happen immediately following the peak, but a while later. Macro-cracks in Case 1 nucleate and extend gradually, but those in Case 2 form suddenly once the peak load has been reached.

In summary, the newly proposed model can reproduce more key features of tensile failures in quasi-brittle media like concrete [40], and can significantly overcome the over-brittle issue that the lattice models with viscosity neglected are subjected to.

\subsection{Strain-rate sensitivity}

By analyzing Cases $1-5$, the dependence of failure behavior on loading rate is discussed. In this study, we take $\Delta t_{v i}$ as a material-dependent parameter to measure viscosity. In Cases 1-5, the material is kept the same by fixing $\Delta t_{v i}$, thus the loading rate sensitivity can be represented by the variation of $\bar{v} \cdot \Delta t_{\nu 1}$. The results obtained are shown in Fig. 17. The following trends can be observed:

- The linear elastic stage is independent of the loading rate and it ends when the controlled displacement reaches $5.43 \mu \mathrm{m}$.

- The higher the loading rate $\bar{v}$, the longer the pre-peak nonlinear stage is , and the higher the peak load is.

- The crack pattern also strongly depends on the loading rate. In Figs. 16(a) and17(a), at the same level of controlled displacement, the accumulated number of broken elements increases with increasing loading rate. This can be explained as follows. In the case of a higher loading rate, the controlled displacement increases faster such that a bigger increment of displacement would occur when each VF field has finished releasing. From Figs. 16(b)-(d) and17(b)-(d), the difference in loading rate leads to very different final crack patterns. For example, a single macro-crack mainly appears in Cases 2 and 3, while two or more macro-cracks happen in other cases.

- The post-peak parts of the force-displacement curves become more and more ductile with increasing loading rate, indicated by curves of failed element number versus displacement in Fig. 17(a).

We have also simulated the loading rate switching problem, i.e. Case 6. As shown in Fig. 18, due to the loading-rate shifting, the force-displacement curve, the evolution of broken element number and the final crack pattern deviate from both Cases 1 and 3. Before the rate has been switched from $\bar{v}=5.0 \times 10^{-5} \mathrm{~m} / \mathrm{s}$ to $\bar{v}=5.0 \times 10^{-7} \mathrm{~m} / \mathrm{s}$, each stored VF field takes an increment in controlled displacement, $7.5 \times 10^{-6} \mathrm{~m}$, to release. However, when the rate is $\bar{v}=5.0 \times 10^{-7} \mathrm{~m} / \mathrm{s}$, it only takes $7.5 \times 10^{-8} \mathrm{~m}$. This explains why the force-displacement curve becomes less ductile under a lower loading rate.

\subsection{Post-peak softening caused by viscosity}

To study the dependence of failure property on viscosity, there is a need to adjust the viscosity-related parameter $\Delta t_{v i}$ under a fixed loading rate $\bar{v}$.

Before everything, it is worth noting a property of the present method. In Cases $1-5$, we have fixed $\Delta t_{v 1}=0.01 \mathrm{~s}$, so a larger $\tilde{d}$ corresponds to a larger loading rate $\bar{v}$ according to $\bar{v} \cdot \Delta t_{v 1}=\tilde{d}$. However, if we fix the value of $\bar{v}$, the change in $\tilde{d}$ will be purely due to the change in $\Delta t_{v 1}$. Since the failure behavior of the present model is governed by the value of $\tilde{d}$, we decide to consider the following equivalent relations:

Case 1: $\bar{v}=5.0 \times 10^{-5} \mathrm{~m} / \mathrm{s}, \Delta t_{v 1}=0.01 \mathrm{~s} \Leftrightarrow \bar{v}=5.0 \times 10^{-5} \mathrm{~m} / \mathrm{s}, \Delta t_{v 1}=0.01 \mathrm{~s}$.

Case 2: $\bar{v}=5.0 \times 10^{-15} \mathrm{~m} / \mathrm{s}, \Delta t_{v 1}=0.01 \mathrm{~s} \Leftrightarrow \bar{v}=5.0 \times 10^{-5} \mathrm{~m} / \mathrm{s}, \Delta t_{v 1}=1.0 \times 10^{-12} \mathrm{~s}$

Case 3: $\bar{v}=5.0 \times 10^{-7} \mathrm{~m} / \mathrm{s}, \Delta t_{v 1}=0.01 \mathrm{~s} \Leftrightarrow \bar{v}=5.0 \times 10^{-5} \mathrm{~m} / \mathrm{s}, \Delta t_{v 1}=1.0 \times 10^{-4} \mathrm{~s}$.

Case 4: $\bar{v}=5.0 \times 10^{-6} \mathrm{~m} / \mathrm{s}, \Delta t_{v 1}=0.01 \mathrm{~s} \Leftrightarrow \bar{v}=5.0 \times 10^{-5} \mathrm{~m} / \mathrm{s}, \Delta t_{v 1}=1.0 \times 10^{-3} \mathrm{~s}$

Case 5: $\bar{v}=5.0 \times 10^{-4} \mathrm{~m} / \mathrm{s}, \Delta t_{v 1}=0.01 \mathrm{~s} \Leftrightarrow \bar{v}=5.0 \times 10^{-5} \mathrm{~m} / \mathrm{s}, \Delta t_{v 1}=0.1 \mathrm{~s}$.

Thus, the obtained results for Cases 1-5, i.e. Fig. 17, can also be used for parameter study related to viscosity, which is represented by $\Delta t_{v 1}$. A larger $\Delta t_{v 1}$ means that the VF field takes a longer time to release, which generally leads to a more 


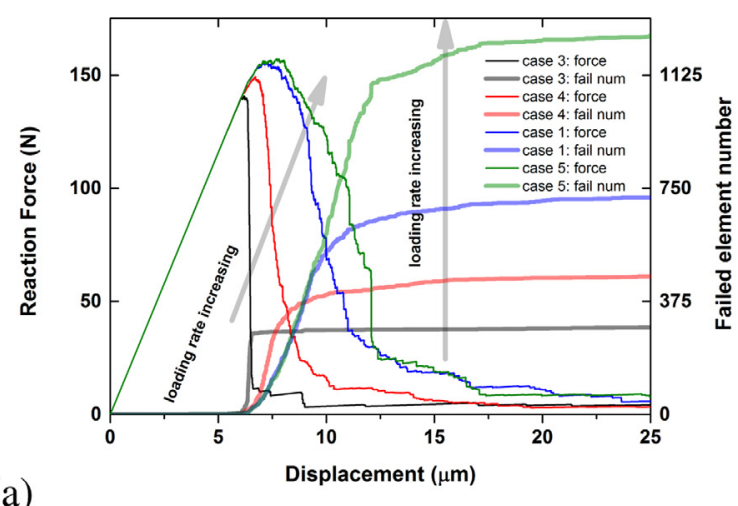

(a)

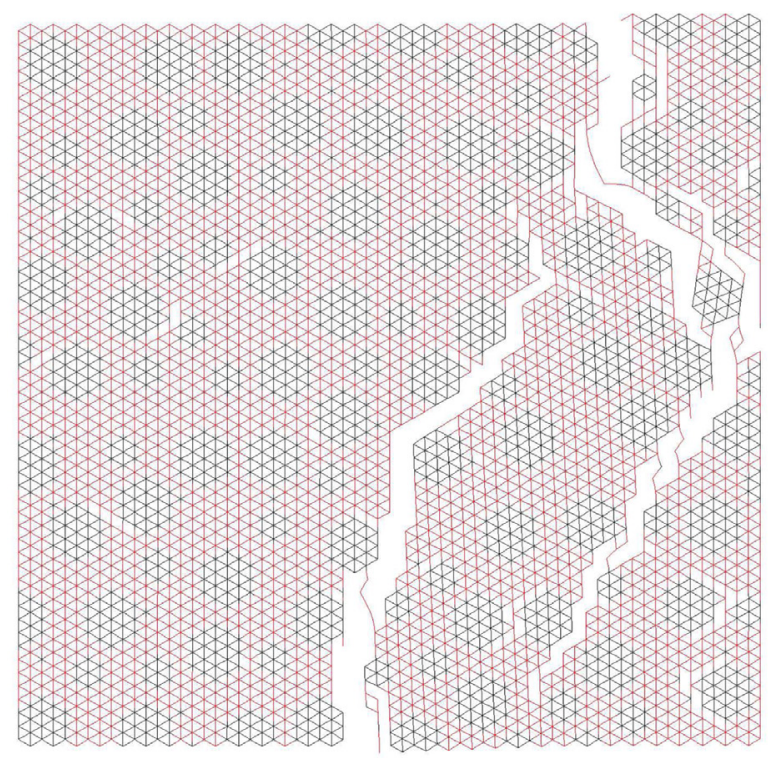

(c)

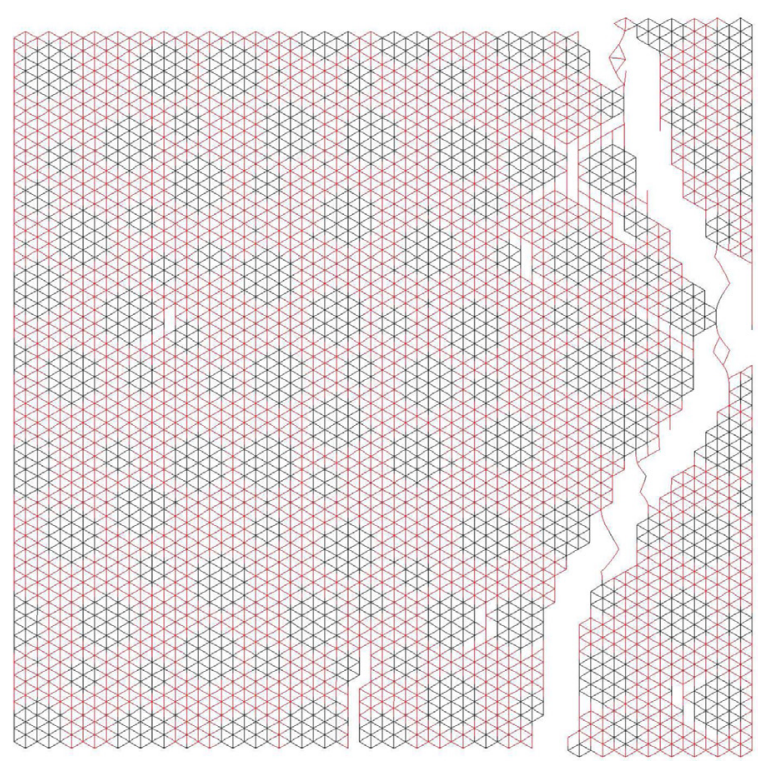

(b)

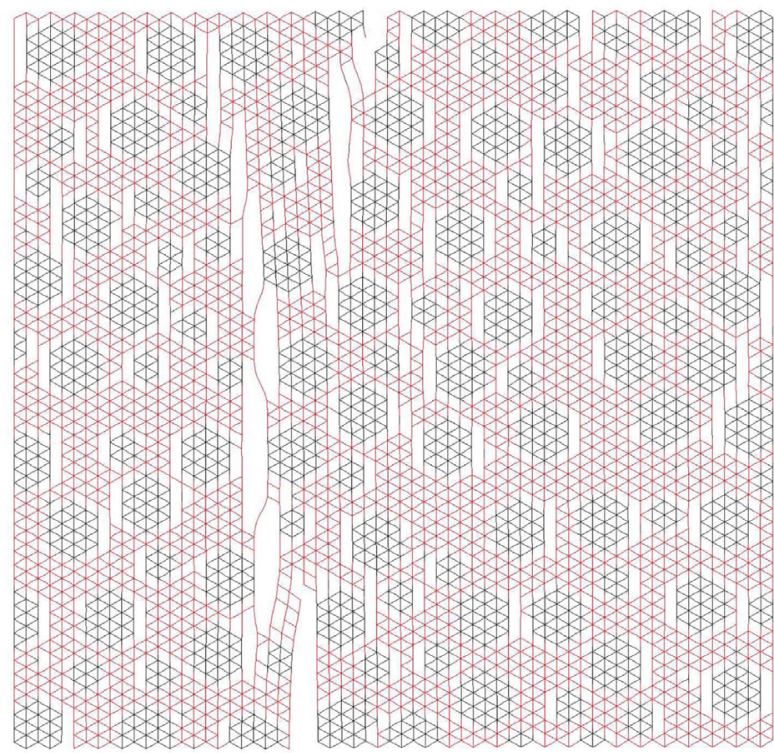

(d)

Fig. 17. Loading rate sensitivity illustrated by Cases 1-5. (a) Diagram of reaction force and accumulated broken element number versus the controlled displacement and crack patterns of (b) Case 3, (c) Case 4 and (d) Case 5.

profound ductility. Particularly, the post-peak ductility is usually described as softening. Therefore, we conclude that the present model helps to explain how the material softening is produced based on the VF-releasing mechanism.

Again, the overbrittle post-peak issue remains an open question in the lattice modeling of quasi-brittle materials $[11,12,20,21]$. It is interesting to note from Fig. 17 that that the post-peak ductility can be recurred largely via considering the viscous effect associated with the release of VF fields induced by elemental breakages, and that most of previous lattice models can be taken as one extremity of the present model, which is the particular case where viscosity is neglected.

\subsection{Immediate elasticity versus viscosity}

When a material is subjected to a sudden load, partial deformation happens simultaneously, which is called immediate elasticity. While the remaining deformation takes some time to arise. If we take the internal force to be released in a breaking element as a sudden load to the specimen, a part of it causes immediate deformation, and the other produces 


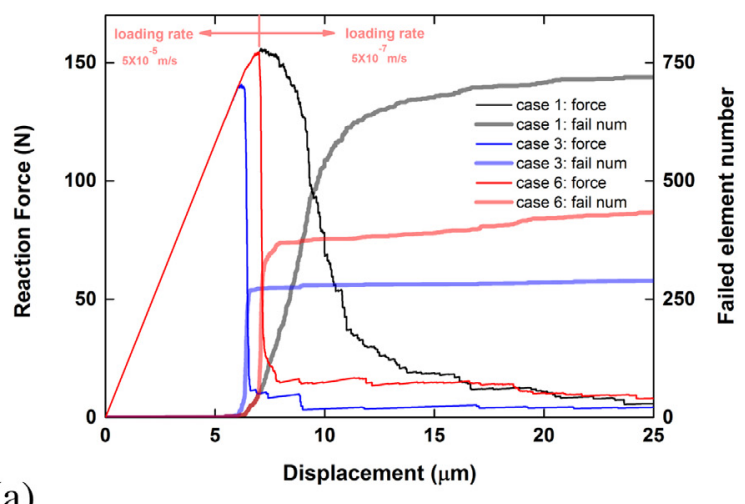

(a)

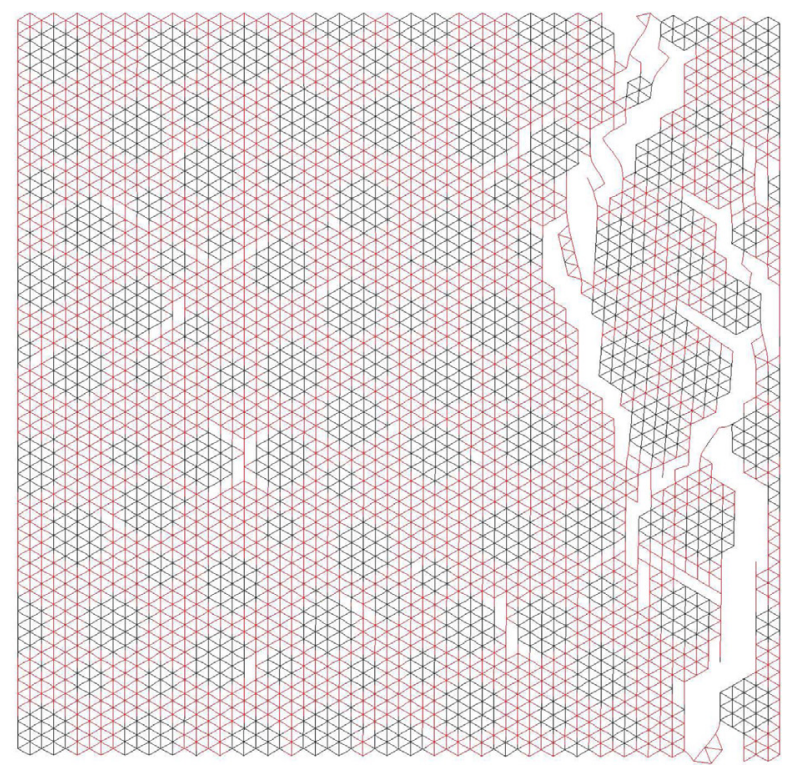

(b)

Fig. 18. Study of loading rate switching problem by comparing Case 6 with Cases 1 and 3. (a) Diagram of reaction force and accumulated broken element number versus controlled displacement, and (b) the final crack pattern of Case 6.

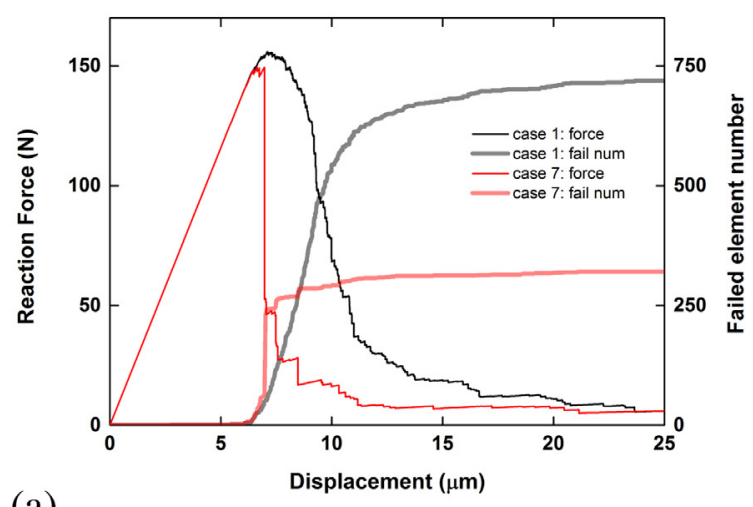

(a)

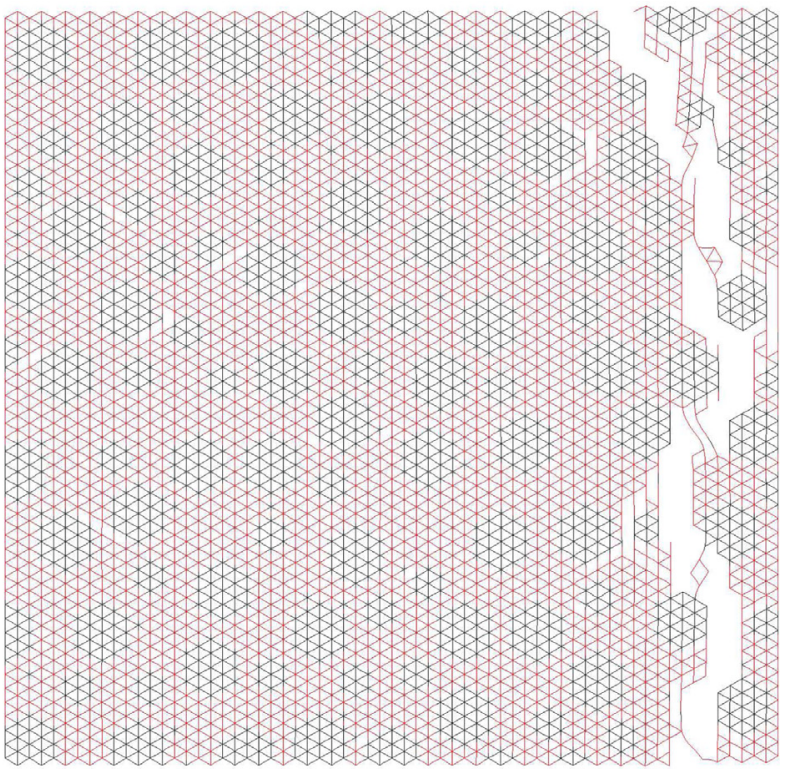

(b)

Fig. 19. Influence of immediate elasticity, analyzed by comparing Case 7 with $\Delta \bar{V}_{1} / \bar{V}=0.6$ and Case 1 with $\Delta \bar{V}_{1} / \bar{V}=0.2$. (a) Diagram of reaction force and accumulated broken element number versus the controlled displacement, and (b) the final crack pattern of Case 7.

viscous deformation. The relative proportion of immediate release is noted by $\Delta \bar{V}_{1} / \bar{V}$, where the symbols of $\Delta \bar{V}_{1}$ and $\bar{V}_{1}$ have been defined in Section 3 and will not be reiterated here. Case 7 with $\Delta \bar{V}_{1} / \bar{V}=0.6$ is compared with Case 1 with $\Delta \bar{V}_{1} / \bar{V}=0.2$, as shown in Fig. 19. A more significant role played by the immediate elasticity would lead to a lower peak, a steeper fall right after the peak and a much weaker ductility. In the extreme case where $\Delta \bar{V}_{1} / \bar{V}=1$, namely when the whole deformation is purely immediately elastic, the present model is reduced to the previous lattice models which have the weakness of unrealistic brittleness. 


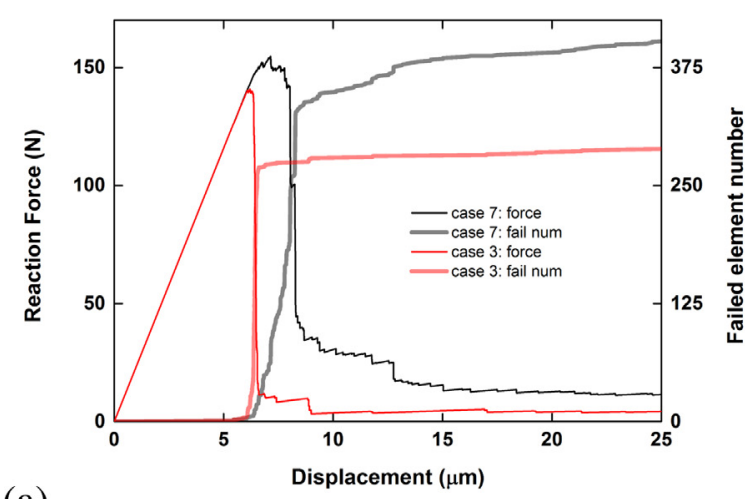

(a)

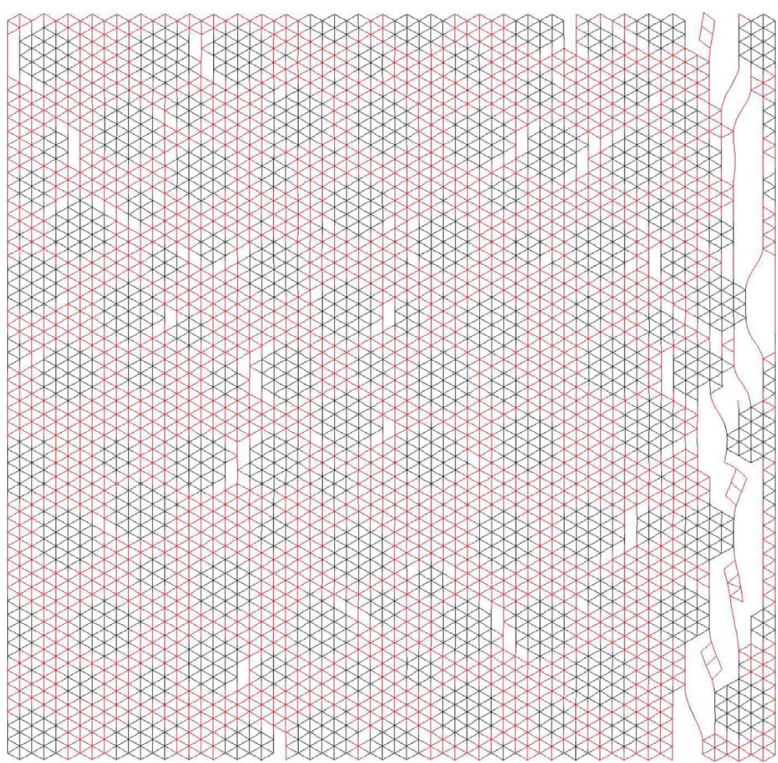

(b)

Fig. 20. Influence of permanent deformation, analyzed by comparing Cases 8 and 1. (a) Diagram of reaction force and accumulated broken element number versus the controlled displacement, and (b) the final crack pattern of Case 8.

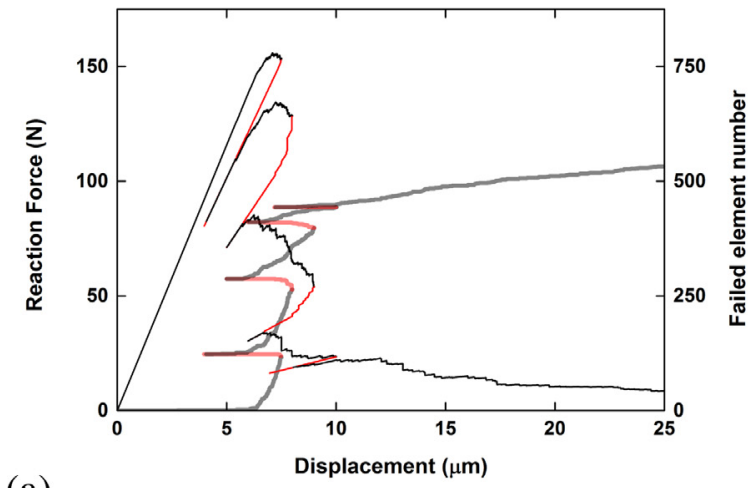

(a)

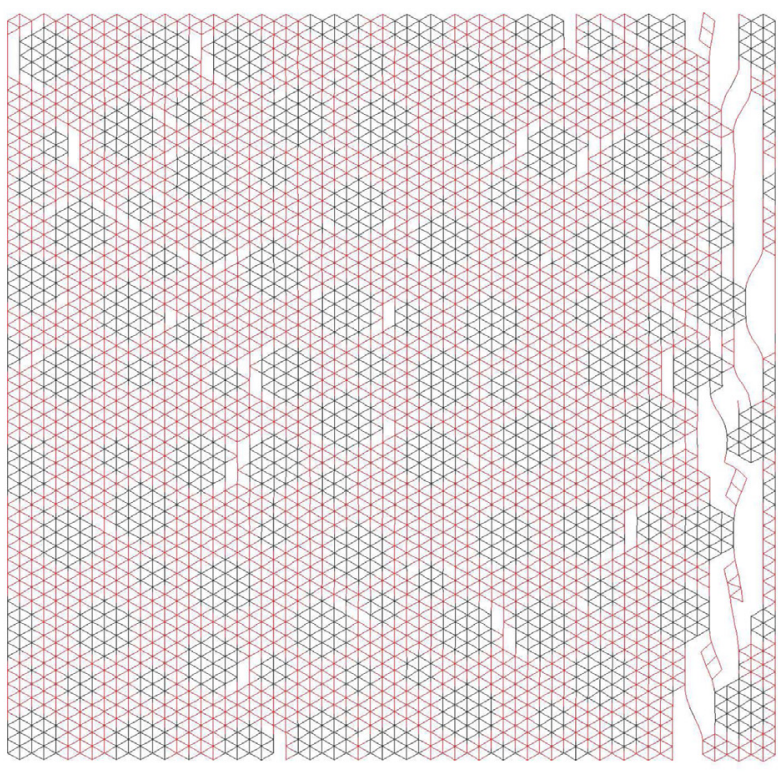

(b)

Fig. 21. Failure behavior under cyclic loading, analyzed by comparing Cases 9 and 1. (a) Diagram of reaction force (thin line) and accumulated broken element number (thick line) versus the controlled displacement, and (b) the final crack pattern of Case 9.

\subsection{Role of permanent deformation}

In the proposed model, we assume that the VF fields take a series of time increments $\Delta t_{v i}$ to release. If a particular time increment $\Delta t_{v i}$ is set to be far larger than the testing time scale, the VF field corresponding to that time increment, i.e. $\Delta \bar{V}_{i}$ will not be released during the interested time range, resulting that the corresponding part of deformation would not recover.

In Case $8, \Delta t_{v i}(i=2,3,4)$ is set as $10^{8} \Delta t_{v 1}$, which indicates that the last $60 \%$ of the viscous force field will not be released within the interested time range which is far smaller than $10^{8} \Delta t_{v 1}$, leading to permanent deformation. Fig. 20 shows the corresponding results. When compared with Case 3 where there is no permanent deformation, a higher peak load is 
achieved. Although there is also a steep fall in the displacement-force curve after the peak, the post-peak response becomes somehow more ductile. Furthermore, the failure becomes more scattering, i.e. more elemental breakages happen. For example, when the controlled displacement is equal to $25 \mu \mathrm{m}$, the total number of elemental failures in Case 8 is 406 , which is much larger than that in Case 3, i.e. 289. As mentioned above, the influence of each elemental breakage to the whole system is attributed to two aspects: one is the degradation of stiffness, and the other is the induction of further failure by releasing its internal force and VF fields. In Case 8, the latter kind of influence becomes much weaker because the VF fields are only partially released within the interested time. As a result, induced failures are harder to occur, which delays the occurrence of macroscopic localized cracks.

\subsection{Cyclic response}

In Case 9, the specimen fails under cyclic loading, as shown in Fig. 21. In the previous lattice models where viscous effects are neglected, the response during the unloading stage is purely linear elastic without occurrence of new failures as long as the controlled displacement has not changed the sign, and the unloading stiffness is equal to the secant stiffness. However, in Case 9, the unloading processes are nonlinear and the elemental breakages continue to arise, due to the fact that the VF fields continue to release with the elapse of time whether it is loading or unloading stage. Furthermore, the unloading stiffness significantly deviates from the secant stiffness. Apparently, the above phenomenon illustrated in Case 9 coincides with some existing experimental observations [12,41]. We have examined that in Case 2, if the cyclic loading setting used here is adopted, both the force-displacement curve and the final crack pattern would remain the same as their monotonic loading counterparts presented in Fig. 16(a) and (d). However, the final crack pattern obtained in Case 9 becomes very different from that presented in Fig. 16(d).

\section{Conclusions}

We have developed a procedure to simulate progressive failure processes in quasi-brittle media such as concrete and rock. As a main modification to previous lattice models [21,37,42], temporal damage evolution has been considered by incorporating the characteristic time for the release of viscous force fields. Firstly, the storage of VF fields is implemented by releasing the internal forces of the critical element. Subsequently, a stepwise algorithm is proposed to deal with the time-dependent stress redistribution induced by the release of VF field release, which has succeeded in excluding any nonlinear iterations. The present model, like the non-iterative sequentially linear algorithm [35], is capable of simulating failure processes in non-proportional loading problems.

By simulating uniaxial tensile tests, the present method has successfully illustrated its following abilities:

- It exhibits the post-peak softening behaviors reasonably well, and helps to achieve a better understanding about the unreasonably overbrittle problem, which exists in many previous lattice models that have no temporal effects. In the proposed model, the post-peak softening and the temporal stress-redistribution mechanism are coupled naturally.

- It reproduces the rate-sensitivity of quasi-brittle failures. Notably, this capability is achieved without the expense of introducing any nonlinear iteration calculations. Instead, the progressive failure is tracked by solving pure linear equation systems based on the principle of superposition.

- The effect of permanent deformation can be easily implemented by simply delaying the release of partial or whole VF fields.

- Besides, the concept of VF field release can be helpful in understanding highly complex catastrophic failures. For example, after a principal earthquake, some aftershocks may follow in the next few minutes, hours or days. This may be explained as follows: the main shock causes cracks, hence, the internal forces previously kept on the potential crack surfaces are released into the surrounding media, with a VF field stored simultaneously. Subsequently, such a VF field which is essentially due to the main shock will be released gradually, and can possibly induce further cracks, i.e. aftershocks.

Notably, "viscous force", which is the core concept introduced in the present study, is different from inertia. On one hand, viscosity is associated with the temporal adjustment of material structure, while inertia arises from acceleration of material points. On the other hand, numerical strategies to deal with them are quite different. Viscous force is considered as static, e.g. in calculating the incremental displacement field caused by VF relaxation as shown in Fig. 13. However, such a storage-relaxation algorithm for VF does not work in the case of inertia. The VF here should not be considered as the inertia force.

Corresponding to the VF-field concept, the key parameter newly introduced is $\alpha$, which is material-dependent and measures the proportions of immediate elastic and viscous deformations induced by a local breakage. When compared the present method with that in [17], even though both of them have the capability of dealing with non-proportional loading problems, some differences exist. (1) In the latter, the counterpart of $\alpha$ is $\omega$, which has a broad range to choose value and can be even changed after every rupture occurring during the relaxation process. (2) The constitutive law in [17] is force-deformation, and here it is force-time (Fig. 11).

Besides, the stepwise constitutive approximation adopted in this study may be replaced by the piece-wise linear one. This means that, for example, in Figs. 6 and 12, the saw-tooth $A \rightarrow B \rightarrow C$ can be replaced by a smooth linear process. 


\section{Acknowledgements}

The work of J.X. Liu was supported by the National Natural Science Foundation of China (Grant No. 11672119), Jiangsu Science Fund for Youth (BK20140520), the Jiangsu University and Jiangsu Specially-Appointed Professor grants. A.K. Soh was supported by the 2017 Monash University Malaysia Strategic Large Grant Scheme (Project code: LG-2017-04-ENG) and the Advanced Engineering Programme, Monash University Malaysia. The authors are indebted to the Editor and reviewers for their highly constructive suggestions.

\section{References}

[1] C.A. Tang, Numerical simulation of progressive rock failure and associated seismicity, Int. J. Rock Mech. Min. Sci. 34 (2) (1997) $249-261$.

[2] D. Potyondy, P. Cundall, A bonded-particle model for rock, Int. J. Rock Mech. Min. Sci. 41 (8) (2004) 1329-1364.

[3] M.D. Wei, F. Dai, N.W. Xu, Y. Xu, K. Xia, Three-dimensional numerical evaluation of the progressive fracture mechanism of cracked chevron notched semi-circular bend rock specimens, Eng. Fract. Mech. 134 (2015) 286-303.

[4] D. Xenos, D. Gregoire, S. Morel, P. Grassl, Calibration of nonlocal models for tensile fracture in quasi-brittle heterogeneous materials, J. Mech. Phys. Solids 82 (2015) 48-60.

[5] H.P. Song, H. Zhang, D.H. Fu, Q. Zhang, Experimental analysis and characterization of damage evolution in rock under cyclic loading, Int. J. Rock Mech. Min. Sci. 88 (2016) 157-164.

[6] S.Y. Wang, S.W. Sloan, D.C. Sheng, C.A. Tang, 3D numerical analysis of crack propagation of heterogeneous notched rock under uniaxial tension, Tectonophysics 677-678 (2016) 45-67.

[7] D. Krajcinovic, M. Basista, Rupture of central-force lattices, J. Phys. 1 (1991) 241-245.

[8] S. Mastilovic, D. Krajcinovic, Statistical models of brittle deformation. part II: computer simulations, Int. J. Plast. 15 (4) (1999) 427-456.

[9] J.G.M. van Mier, M.R.A. van Vliet, T.K. Wang, Fracture mechanisms in particle composites: statistical aspects in lattice type analysis, Mech. Mater. 34 (11) (2002) 705-724.

[10] M. Ostoja-Starzewski, Lattice models in micromechanics, Appl. Mech. Rev. 55 (1) (2002) 35-60.

[11] B.L. Karihaloo, P.F. Shao, Q.Z. Xiao, Lattice modelling of the failure of particle composites, Eng. Fract. Mech. 70 (17) (2003) 2385-2406

[12] A. Rinaldi, Y.C. Lai, Statistical damage theory of 2D lattices: energetics and physical foundations of damage parameter, Int. J. Plast. 23 (10-11) (2007) $1796-1825$.

[13] J.X. Liu, S.C. Deng, J. Zhang, N.G. Liang, Lattice type of fracture model for concrete, Theor. Appl. Fract. Mech. 48 (3) (2007) $269-284$.

[14] P. Grassl, C. Fahy, D. Gallipoli, S.J. Wheeler, On a 2D hydro-mechanical lattice approach for modelling hydraulic fracture, J. Mech. Phys. Solids 75 (2015) 104-118.

[15] Q. Ma, H.Y. Cheng, K.I. Jang, H.W. Luan, K.C. Hwang, J.A. Rogers, Y.G. Huang, Y.H. Zhang, A nonlinear mechanics model of bio-inspired hierarchical lattice materials consisting of horseshoe microstructures, J. Mech. Phys. Solids 90 (2016) 179-202.

[16] J.E. Bolander, S. Saito, Fracture analyses using spring networks with random geometry, Eng. Fract. Mech. 61 (5-6) (1998) 569-591

[17] J. Eliáš, Generalization of load-unload and force-release sequentially linear methods, Int. J. Damage Mech. 24 (2) (2015) 279-293.

[18] A. Hansen, S. Roux, Statistics Toolbox for Damage and Fracture, Springer, Wien, 2000.

[19] Z.P. Bažant, B.H. Oh, Crack band theory for fracture of concrete, Matér. Constr. 16 (3) (1983) 155-177.

[20] J.X. Liu, T.E. Sayed, On the Load-Unload (L-U) and Force-Release (F-R) algorithms for simulating brittle fracture processes via lattice models, Int. J. Damage Mech. 21 (7) (2012) 960-988.

[21] G. Lilliu, J.G.M. van Mier, 3D lattice type fracture model for concrete, Eng. Fract. Mech. 70 (7-8) (2003) 927-941.

[22] J.G.M. van Mier, Lattice modelling of size effect in concrete strength by Ince R, Arslan A, Karihaloo BL (Engineering Fracture Mechanics (2003) 70), Eng. Fract. Mech. 71 (11) (2004) 1625-1628.

[23] W. Alnaas, Nonlinear Finite Element Analysis of Quasi-Brittle Materials, Cardiff University, 2016 Ph.D. thesis.

[24] J.G.M. van Mier, Concrete Fracture: A Multiscale Approach, CRC Press, 2012.

[25] Y.L. Bai, H.Y. Wang, M.F. Xia, F.J. Ke, Statistical mesomechanics of solid, linking coupled multiple space and time scales, Appl. Mech. Rev. 58 (6) (2005) $286-305$.

[26] D. Krajcinovic, A. Rinaldi, Thermodynamics and statistical physics of damage processes in quasi-ductile solids, Mech. Mater. 37 (2-3) (2005) 299-315.

[27] J. Li, X.D. Ren, Multi-scale based stochastic damage evolution, Eng. Fail. Anal. 18 (2) (2011) 726-734.

[28] J. Rots, B. Belletti, S. Invernizzi, Robust modeling of RC structures with an "event-by-event" strategy, Eng. Fract. Mech. 75 (3-4) (2008) $590-614$.

[29] M.A. Crisfield, Nonlinear Finite Element Analysis of Solids and Structures, Wiley, 2012.

[30] J. Li, J.Y. Wu, J.B. Chen, Stochastic Damage Mechanics of Concrete Structures (in Chinese), Science Press, 2014.

[31] G. Cusatis, Strain-rate effects on concrete behavior, Int. J. Impact Eng. 38 (4) (2011) 162-170.

[32] S.X. Wu, X.D. Chen, J.K. Zhou, Tensile strength of concrete under static and intermediate strain rates: correlated results from different testing methods, Nucl. Eng. Des. 250 (2012) 173-183.

[33] X.L. Du, L. Jin, G.W. Ma, Numerical simulation of dynamic tensile-failure of concrete at meso-scale, Int. J. Impact Eng. 66 (2014) 5-17.

[34] H. Xu, H.M. Wen, A computational constitutive model for concrete subjected to dynamic loadings, Int. J. Impact Eng. 91 (2016) 116-125.

[35] J. Eliáš, P. Frantik, M. Vorechovsky, Improved sequentially linear solution procedure, Eng. Fract. Mech. 77 (12) (2010) 2263-2276.

[36] R. Costa, J. Alfaiate, D. Dias-da Costa, L. Sluys, A non-iterative approach for the modelling of quasi-brittle materials, Int. J. Fract. 178 (1-2) (2012) $281-298$.

[37] J.X. Liu, Z.Y. Zhao, S.C. Deng, N.G. Liang, Numerical investigation of crack growth in concrete subjected to compression by the generalized beam lattice model, Comput. Mech. 43 (2) (2009) 277-295.

[38] A. Ibrahimbegovic, A. Delaplace, Microscale and mesoscale discrete models for dynamic fracture of structures built of brittle material, Comput. Struct. 81 (12) (2003) 1255-1265.

[39] J.X. Liu, Z.Y. Zhao, S.C. Deng, N.G. Liang, A simple method to simulate shrinkage-induced cracking in cement-based composites by lattice-type modeling, Comput. Mech. 43 (4) (2009) 477-492.

[40] E.P. Prado, J.G.M. van Mier, Effect of particle structure on mode i fracture process in concrete, Eng. Fract. Mech. 70 (14) (2003) $1793-1807$.

[41] Z.J. Yang, D. Proverbs, A comparative study of numerical solutions to non-linear discrete crack modelling of concrete beams involving sharp snap-back, Eng. Fract. Mech. 71 (1) (2004) 81-105.

[42] A. Rinaldi, D. Krajcinovic, P. Peralta, Y.C. Lai, Lattice models of polycrystalline microstructures: a quantitative approach, Mech. Mater. 40 (1-2) (2008) $17-36$. 